\title{
High-resolution PIV measurements of a transitional shock wave-boundary layer interaction
}

\author{
R. H. M. Giepman ${ }^{1}$ F. F. J. Schrijer ${ }^{1}$ B. W. van Oudheusden ${ }^{1}$
}

Received: 11 February 2015 / Revised: 22 April 2015 / Accepted: 23 April 2015 / Published online: 22 May 2015

(C) The Author(s) 2015. This article is published with open access at Springerlink.com

\begin{abstract}
This study investigates the effects of boundary layer transition on an oblique shock wave reflection. The Mach number was 1.7, the unit Reynolds number was $35 \times 10^{6} \mathrm{~m}^{-1}$, and the pressure ratio over the interaction was 1.35 . Particle image velocimetry is used as the main flow diagnostics tool, supported by oil-flow and Schlieren visualizations. At these conditions, the thickness of the laminar boundary layer is only $0.2 \mathrm{~mm}$, and seeding proved to be problematic as practically no seeding was recorded in the lower $40 \%$ of the boundary layer. The top $60 \%$ could, however, still be resolved with good accuracy and is found to be in good agreement with the compressible Blasius solution. Due to the effects of turbulent mixing, the near-wall seeding deficiency disappears when the boundary layer transitions to a turbulent state. This allowed the seeding distribution to be used as an indicator for the state of the boundary layer, permitting to obtain an approximate intermittency distribution for the boundary layer transition region. This knowledge was then used for positioning the oblique shock wave in the laminar, transitional (50\% intermittency) or turbulent region of the boundary layer. Separation is only recorded for the laminar and transitional interactions. For the laminar interaction, a large separation bubble is found, with a streamwise length of $96 \delta_{i, 0}^{*}$. The incoming boundary layer is lifted over the separation bubble and remains in a laminar state up to the impingement point of the shock wave. After the shock, transition starts and a turbulent profile is reached approximately $80-90 \delta_{i, 0}^{*}$
\end{abstract}

\section{R. H. M. Giepman \\ R.H.M.Giepman@tudelft.nl}

1 Aerospace Engineering Department, Delft University of Technology, Kluyverweg 1, 2629 HS Delft, The Netherlands downstream of the shock. Under the same shock conditions, the transitional interaction displays a smaller separation bubble $\left(43 \delta_{i, 0}^{*}\right)$, and transition is found to be accelerated over the separation bubble.

\section{Introduction}

Shock wave-boundary layer interactions (SWBLI) can be encountered in a wide range of applications that are relevant for the high-speed flight regime, such as supersonic intakes, the blade passages of jet engines and transonic wings. These interactions can generate undesirable features, such as increased pressure losses, flow unsteadiness and localized heat loads. Many of these features are associated with the occurrence of flow separation (Dolling 2001). The two dominant parameters determining the severity of the interaction are the strength of the shock wave and the state of the incoming boundary layer (laminar/transitional/ turbulent). Since turbulent interactions are more often found in flight conditions (Babinsky and Harvey 2011), most researchers have focussed their attention to this particular type of interaction. However, at high altitudes and thus low Reynolds numbers, it is not unlikely that parts of the boundary layer in, for instance, a jet engine are still in a laminar/transitional state (Mayle 1991).

It is well known that laminar boundary layers are much more prone to separation than turbulent boundary layers. Gadd et al. (1954) were among the first workers to experimentally investigate laminar, transitional and turbulent interactions. From Schlieren and pressure measurements, they found that the laminar interaction exhibits a large and flat separation bubble which extends both far upstream and downstream of the impinging shock wave. The separation bubble has a triangular shape and acts as a ramp for 
the incoming flow, creating a series of weak compression waves upstream of the incident shock wave. The boundary layer is lifted over the bubble and continues nearly unaltered (Hakkinen et al. 1959). The incident shock reflects as an expansion wave from the top of the separation bubble shear layer, and at reattachment, again a series of compression waves is created.

For weak shock waves and low Reynolds number, it is possible for the boundary layer to remain laminar throughout the interaction region. The work of Le Balleur and Délery (1973), however, showed that the transition front moves gradually upstream with the shock strength. For pressure jumps larger than 1.4, they found that, independent of the Reynolds number, transition sets in at the impact point of the incident shock. A mixed type of interaction is therefore obtained, with a laminar boundary layer upstream of the shock and the downstream part of the interaction exhibiting a transitional behaviour. This transitional behaviour of the boundary layer results in increased mixing and supports the boundary layer to overcome the pressure rise at reattachment, therefore reducing the size of the separation bubble. The size of the separation bubble shows a near-linear dependence with the driving pressure coefficient (pressure jump minus pressure required for incipient separation) and the local displacement thickness (Gadd et al. 1954; Hakkinen et al. 1959; Katzer 1989). Typically, researchers have recorded only weak Reynolds number effects on the size of the separation bubble (Gadd et al. 1954; Hakkinen et al. 1959; Katzer 1989). For a fully laminar interaction, the separation bubble is found to increase in size with Reynolds number, and for the mixed type of interaction, this trend is reversed.

Our understanding of laminar and transitional shock wave boundary layer interactions has been aided by the continuous development of measurement techniques over the past decades, and the introduction of short-interframetime CCD cameras has made it possible to perform particle image velocimetry (PIV) measurements in both the supersonic and hypersonic regimes (Scarano 2008). In the recent work of Erdem et al. (2013), PIV measurements were performed (among other techniques) on laminar and transitional SWBLI at Mach 5. The impinging shock wave ( $7^{\circ}$ flow deflection angle) separates the incoming boundary layer, and transition is recorded near the end of the separation bubble. The PIV measurements furthermore indicate high turbulence levels in the shear layer between the separation bubble and the freestream. The latter result can only be interpreted qualitatively because of the limited response time of the PIV tracer particles (i.e. a too high Stokes number), which is a common problem when performing PIV in thin supersonic shear layers (Scarano 2008).

Laminar and transitional SWBLIs have also attracted attention from the numerical community. Teramoto (2005) performed LES simulations on the experimental configuration of Hakkinen et al. (1959) for a transitional SWBLI. The results of the LES simulation were in good agreement with the pressure measurements of Hakkinen et al. (1959) and also the recovering boundary layer was captured fairly accurately. From the LES simulations, the formation of a series of low- and high-speed streaks could be identified in the region between the incident shock and the reattachment location. These streaks were accompanied by both longitudinal vortical structures and hairpin vortices, which are thought to play an important role in the transition process.

The receptivity of the laminar separation bubble to outside disturbances was further investigated by Sansica et al. (2014). Their DNS simulations showed the development of Kelvin-Helmholtz vortices on the detached shear layer and also revealed low-frequency unsteadiness present at the separation point, very similar to what has been recorded for turbulent SWBLIs (Clemens and Narayanaswamy 2014).

The particular objective of the current study is to gain a better understanding of the differences between laminar, transitional and turbulent SWBLIs. In particular, the goal is to describe the process of boundary layer transition in the interaction zone for the laminar and transitional interaction. To this end, high-resolution (130 pix/mm) PIV measurements were performed that accurately capture the mean flow field for the different types of interactions. By tracing the development of the shape factor throughout the interaction region, it was possible to unambiguously determine the onset and development of transition. The evidence from the PIV measurements was further supported by oil-flow and high-speed Schlieren visualizations.

Measuring supersonic transitional boundary layers with PIV under these conditions is highly challenging, because of the extremely thin laminar boundary layer $(\sim 0.2 \mathrm{~mm})$, the high shear rates ( $\sim .9 \mathrm{pixel} / \mathrm{pixel})$ and the non-uniform seeding distribution. A second objective of this investigation was therefore to further develop the PIV tools necessary for analysing flows under these difficult circumstances.

Section 2 discusses the experimental set-up that was used. A particular emphasis has been placed here on the description of the freestream fluctuations, which are of crucial importance when characterizing boundary layer transition experiments. Section 3 continues with the processing tools that were used for the analysis of the PIV data, and in Sect. 4, these tools are applied to the PIV images of the undisturbed (no shock) flat-plate boundary layer. Based upon the undisturbed boundary layer results describing the transition location and intermittency, an oblique shock wave was positioned in the laminar, transitional or turbulent part of the boundary layer. Finally, Sect. 5 presents a discussion of the data that were recorded for the three types of interactions. The size and shape of the separation bubble are derived from the PIV data, and the transition process in 
the flow over the bubble is captured by tracing the development of the incompressible shape factor.

\section{Experimental set-up}

\subsection{Tunnel conditions}

The experiments were carried out in the TST-27 blowdown transonic/supersonic wind tunnel of Delft University of Technology. The test section measures $270 \times 280 \mathrm{~mm}^{2}$, and the tunnel was operated at a Mach number of 1.7 , a total pressure $p_{0}$ of $2.3 \mathrm{bar}$, a total temperature $T_{0}$ of $278 \mathrm{~K}$ with a corresponding unit Reynolds number of $35 \times 10^{6} \mathrm{~m}^{-1}$. Due to the emptying of the storage vessel, the total temperature decreases slightly during the course of a run, by approximately $4-5 \mathrm{~K}$ for a typical 30 -s wind tunnel run.

As this study deals with boundary layer transition, which is sensitive to external flow disturbances, a dedicated hotwire anemometry measurement was carried out to quantify the freestream fluctuation level of the tunnel at the given operating conditions. The hot wire was operated at a relatively high overheat ratio of 0.9 , in order to make sure it is mostly sensitive to fluctuations in the mass flux $(\rho u)^{\prime}$ and not to fluctuations in the total temperature (Bestion et al. 1983). From the measurements, it followed that the RMS level of the mass flux equals $7.7 \times 10^{-3} \rho_{\infty} u_{\infty}$.

The measured mass flux fluctuations can be converted to pressure fluctuations under the assumption that the disturbances in the tunnel are purely acoustic (Laufer 1964), which is a reasonable assumption for a non-quiet supersonic wind tunnel (Laufer 1961). Following the approach of Laufer (1964), it then follows that the RMS level of the pressure fluctuations equals $1.9 \times 10^{-2} p_{\infty}$.

To further investigate the nature of the freestream disturbances, the shape of the spectrum of the pressure fluctuations has been compared to the spectra measured in the works of Laufer (1964) and Duan et al. (2014) (see Fig. 1). Duan et al. (2014) performed DNS simulations on a supersonic $(M=2.5)$ turbulent boundary layer and analysed the radiated acoustic pressure field. Laufer (1964) performed hot-wire measurements in the freestream of a $M=2$ supersonic wind tunnel and converted the measured mass flux fluctuations to pressure fluctuations. Figure 1 presents the normalized power spectral density. The data of Laufer (1964) and Duan et al. (2014) are not available for $\omega \delta / U_{\infty}<0.2$. Therefore, to allow a fair comparison with the current data set, the spectrum normalization is performed such that the integral over the frequency range $\omega \delta / U_{\infty}>0.2$ equals unity for all three cases. For the current measurements, $p_{\text {rms }}\left(\omega \delta / U_{\infty}>0.2\right)=1.06 \times 10^{-2} p_{\infty}$. The non-dimensional frequency bound of 0.2 corresponds to a dimensional frequency of $0.7 \mathrm{kHz}$. This is based upon

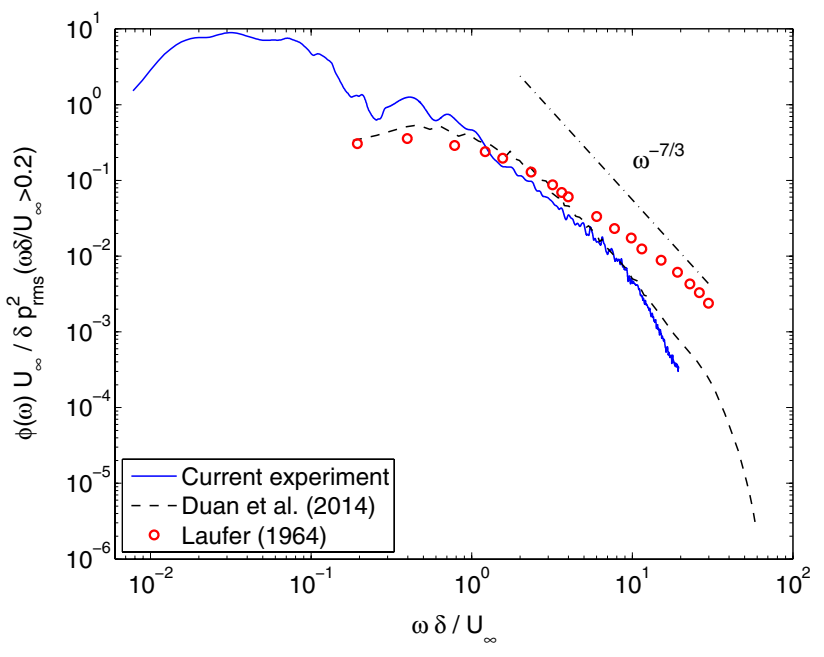

Fig. 1 Energy spectrum of the freestream pressure fluctuations

a freestream velocity of $U_{\infty}=452 \mathrm{~m} / \mathrm{s}$ and a tunnel wall boundary layer thickness of $\delta=2.0 \mathrm{~cm}$.

In the work of Laufer, no exact values were given for the boundary layer thickness $\delta$. It is only mentioned that $\delta$ varies from 2.5 to $5.6 \mathrm{~cm}$ when increasing the freestream Mach number from 1.5 to 5. Duan et al. analysed the data of Laufer and came to the conclusion that the boundary layer thickness should have been $\sim 3.1 \mathrm{~cm}$ for a Mach number of 2. This value has also been used here for presenting the data of Laufer in Fig. 1.

The spectral data of the current experiment are in good agreement with the numerical data of Duan et al. Both data sets display a $\omega^{-7 / 3}$ frequency dependence for $1<\omega \delta /$ $U_{\infty}<10$. For higher frequencies, the data from the current measurement start to deviate from the $-7 / 3$ scaling and start approximating a -4.5 scaling. The data from Duan et al. break away from the $-7 / 3$ scaling for slightly higher frequencies $\left(\omega \delta / U_{\infty}>30\right)$ and then show a -5 scaling. The data from Laufer show a reasonable agreement with the data from the current experiment and the data of Duan et al. in the lower frequency range, but start to deviate for $\omega \delta / U_{\infty}>4$.

Finally, it may be observed that the data of the current experiment show a relatively large low-frequency portion $\left(\omega \delta / U_{\infty}<0.2\right)$, which contains $44 \%$ of all the energy. This low-frequency noise cannot be attributed to the tunnel wall boundary layers and is probably related to a form of valve noise. Although the energy that is contained in this lowfrequency band is not insignificant, it is not expected that it drastically alters the transition behaviour of a flat-plate boundary layer. A stability analysis (De Groot et al. 2014) performed for this particular case shows that the growth rate of the most unstable mode in the laminar boundary layer is four orders of magnitude larger than the growth rate of modes with a frequency of $\omega \delta / U_{\infty}<0.2$. 

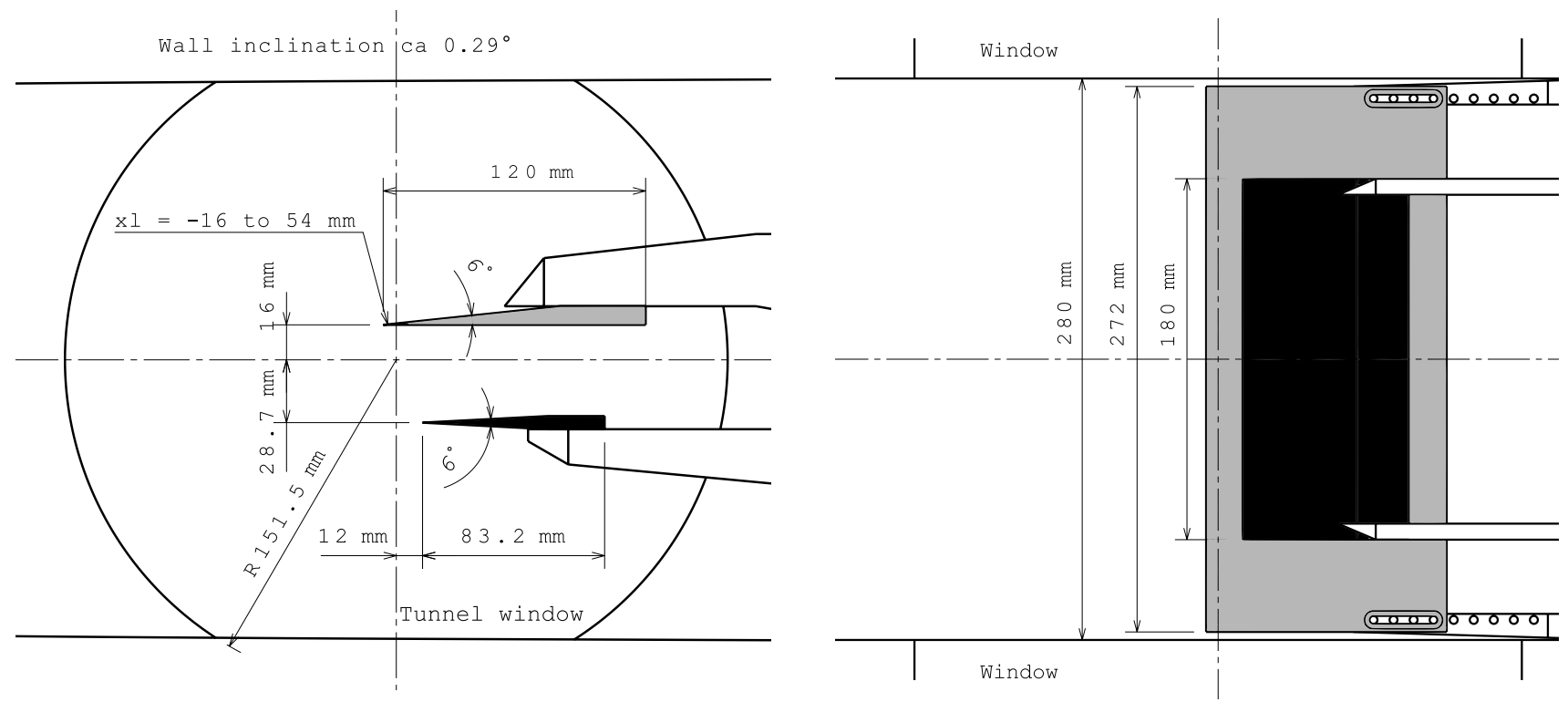

Fig. 2 Side view (left) and bottom view (right) of the wind tunnel configuration (in grey: flat-plate model, in black: shock generator)

\subsection{Wind tunnel models}

The experimental set-up consists of two models, a fullspan flat plate with a sharp leading edge $(R \sim 0.15 \mathrm{~mm})$, which is used to generate a laminar boundary layer and a partial-span shock generator (see Fig. 2). The models can be moved with respect to each other in streamwise direction, which makes it possible to locate the incident shock wave in the laminar, transitional or turbulent region of the boundary layer.

The models were designed to be as slender as possible to limit the amount of blockage and to ensure a steady operation of the wind tunnel at this relatively low supersonic Mach number of 1.7. Schlieren visualizations were used to verify that the tunnel was started properly. Figure 3 shows a case where the oblique shock wave is impinging approximately $31 \mathrm{~mm}$ from the leading edge of the flat plate. The flat plate itself creates a very weak leading edge shock wave $\left(\theta \sim 0.1^{\circ}\right)$, which first reflects on the shock generator and then on the flat plate (sufficiently far downstream of the area of interest).

The shock generator was set to induce a flow deflection angle of $3^{\circ}$, which corresponds to an overall theoretical pressure ratio of 1.35 (based on an inviscid shock reflection). This value can be compared with the incipient separation pressure $p_{\text {inc }}$ as predicted by the free-interaction theory of Chapman et al. (1958):

$\frac{p_{\text {inc }}}{p_{\infty}}=1+C \gamma M_{0}^{2} \sqrt{\frac{2 C_{f 0}}{\left(M_{0}^{2}-1\right)^{0.5}}}$

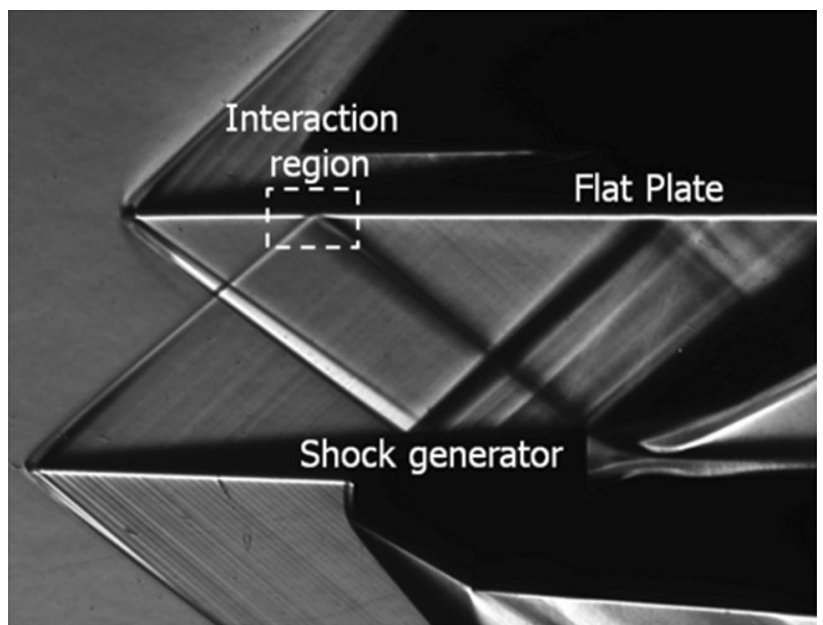

Fig. 3 Schlieren visualization of the wind tunnel configuration

with $M_{0}$ and $C_{f 0}$ the freestream Mach number and skin friction coefficient at the interaction onset, respectively. For laminar interactions, it is found that $C \approx 1$ (Hakkinen et al. 1959) and for turbulent interactions $C \approx 3$ (Babinsky and Harvey 2011). Substitution of the current experimental values in Eq. (1) yields incipient separation pressure ratios of 1.11 and 1.77 for the laminar and turbulent interactions, respectively. It is therefore expected that the pressure ratio of 1.35 that is used for the current experiment leads to a significant portion of reversed flow for the laminar interaction, whereas the turbulent interaction remains attached. 
Table 1 Viewing configuration of the PIV cameras

\begin{tabular}{ll}
\hline Parameter & Value \\
\hline Measurement area & $12.5 \times 5.0 \mathrm{~mm}^{2}$ \\
& $1624 \times 651 \mathrm{pixel}^{2}$ \\
Digital imaging resolution & 130 pixels $/ \mathrm{mm}$ \\
Object focal length & $f=105 \mathrm{~mm}$ \\
$F$-number & $f_{\#}=16$ \\
Laser pulse separation & $430 \mathrm{~ns}$ \\
Freestream particle displacement & 24 pixel \\
\hline
\end{tabular}

\subsection{PIV arrangement}

The PIV measurements were performed with two Lavision Imager LX cameras, placed on either side of the tunnel. The fields of view $\left(12.5 \times 5 \mathrm{~mm}^{2}\right)$ of the two cameras overlap by approximately $3 \mathrm{~mm}$, to allow for the proper recombination of the images. The cameras have a CCD chip of $1624 \times 1236$ pixels, which is cropped in the wall-normal direction from 1236 to 651 pixels to increase the data acquisition rate (acquisition frequency of $10.2 \mathrm{~Hz}$ ). The cameras were equipped with a $105-\mathrm{mm}$ micro-Nikkor lens $\left(f_{\#}=16\right)$, resulting in a magnification of 0.57 and a spatial resolution of 130 pixels $/ \mathrm{mm}$. At this resolution, a $0.2-\mathrm{mm}-$ thick laminar boundary layer (conditions at $x=40 \mathrm{~mm}$ ) is imaged onto 26 pixels. An even higher spatial resolution therefore might seem beneficial; however, increasing the magnification also implies a smaller depth of focus, which has to be compensated for by an increase in the $f_{\# \text { to avoid }}$ out-of-focus particles. The latter has the disadvantage of lower-intensity particles with a larger particle diameter. A trade-off was made between these conflicting requirements, and the presented settings were found to be optimal for these particular measurement conditions.

An important advantage of the cameras that are used is the short interframe time of $430 \mathrm{~ns}$ that can be achieved. At the given spatial resolution, this value results in a freestream particle displacement of $\sim 24$ pixels between two successive frames and a shear ratio of $\sim 0.9$ pixel/pixel for conditions that apply to a laminar boundary layer at $40 \mathrm{~mm}$ from the leading edge. Although this is a relatively high value for the shear ratio [typically it is preferred to keep it below 0.3 (Meunier and Leweke 2003)], the correlation peak can still be constructed with good accuracy. For an overview of the camera settings, see Table 1 .

Illumination is provided by a double-pulse Nd:YAG Spectra Physics Quanta Ray PIV-400 laser, which is operated at a laser power of $140 \mathrm{~mJ}$ per pulse. The pulse duration is $<7 \mathrm{~ns}$, which translates into a particle displacement during illumination of $<0.4$ pixel and therefore introduces negligible particle blur. The flow was seeded with $\mathrm{TiO}_{2}$ particles ( $30 \mathrm{~nm}$ crystal size), which have a response time of $\tau=2.48 \mu$ s after dehydration (the seeding was heated for $40 \mathrm{~min}$ at $120^{\circ} \mathrm{C}$ before usage). In the region of the incident shock wave, this translates into a response length of $\xi=0.7 \mathrm{~mm}$. The response time/length were determined by performing an oblique shock wave test, following the procedure outlined in Ragni et al. (2010).

\section{Data reduction}

\subsection{Pre-processing steps}

\subsubsection{Correction for fixed-pattern camera read-out noise}

The mean noise level of the two megapixel Lavision Imager LX cameras is approximately 150 counts with a random component of 13 counts. Superimposed on this random noise is a constant fixed-pattern noise caused by the progressive scan read-out of the CCD chip. This noise is very weak in individual images (1-3 counts) and exhibits a negligible effect on the correlation peak for single-pair correlation. The situation is, however, different when employing the ensemble correlation technique (Meinhart et al. 2000). The ensemble correlation of constant fixed-pattern noise builds up to yield a coherent component of the correlation whose height is of the order of the correlation peak resulting from the particle displacement.

Figure 4a shows an example of a typical correlation peak without the removal of the fixed-pattern noise. Diagonal lines are present in the correlation plane running from the top-left to the bottom-right part of the image. To estimate the location of the correlation peak with subpixel accuracy, a three-point Gaussian fit is employed, which uses the central peak and its direct neighbours. The accuracy of this fit is deteriorated by the presence of the diagonal lines and consequently so is the accuracy of the estimated velocity vector.

The source of these diagonal lines is expected to be related to the progressive scan read-out process of the camera. Therefore, a dark camera image (no laser or particles) was analysed in the frequency domain to capture the pattern without interference from the particle images (see Fig. 5a). A number of distinct peaks appear in the frequency domain, which were found to coincide with secondary peaks present in the images acquired during the experiment (see Fig. 5b). The peaks are spaced by one-eighth of a wave number in $x$-direction and by one-fourth of a wave number in $y$-direction. It is therefore possible to transform all camera images to the frequency domain, remove the peaks corresponding to the read-out noise and to transform the images back using an inverse Fourier transform. After this processing step, the correlation peak looks much cleaner as can be seen in Fig. $4 \mathrm{~b}$. 
Fig. 4 Correlation peaks obtained by means of the ensemble correlation technique in a turbulent boundary layer approximately $90 \mathrm{~mm}$ from the leading edge of the flat plate at $0.4 \delta_{99}$. a No pre-processing, b read-out noise removed, $\mathbf{c}$ corrected for wall movements, $\mathbf{d}$ min-max-filtered

Fig. 5 Fourier-transformed camera images, presented is the normalized magnitude $|Y| /|Y|_{\max }$ as a function of the wave number in $x$ - and $y$-direction, respectively, $k_{x}$ and $k_{y}$. The red circles mark the read-out noise peaks that were identified in the dark image. a Dark image, b typical PIV image, $\mathbf{c}$ zoom of dark image at peak location $(0.125$, $0.25)$, $\mathbf{d}$ zoom of the PIV image at peak location $(0.125,0.25)$
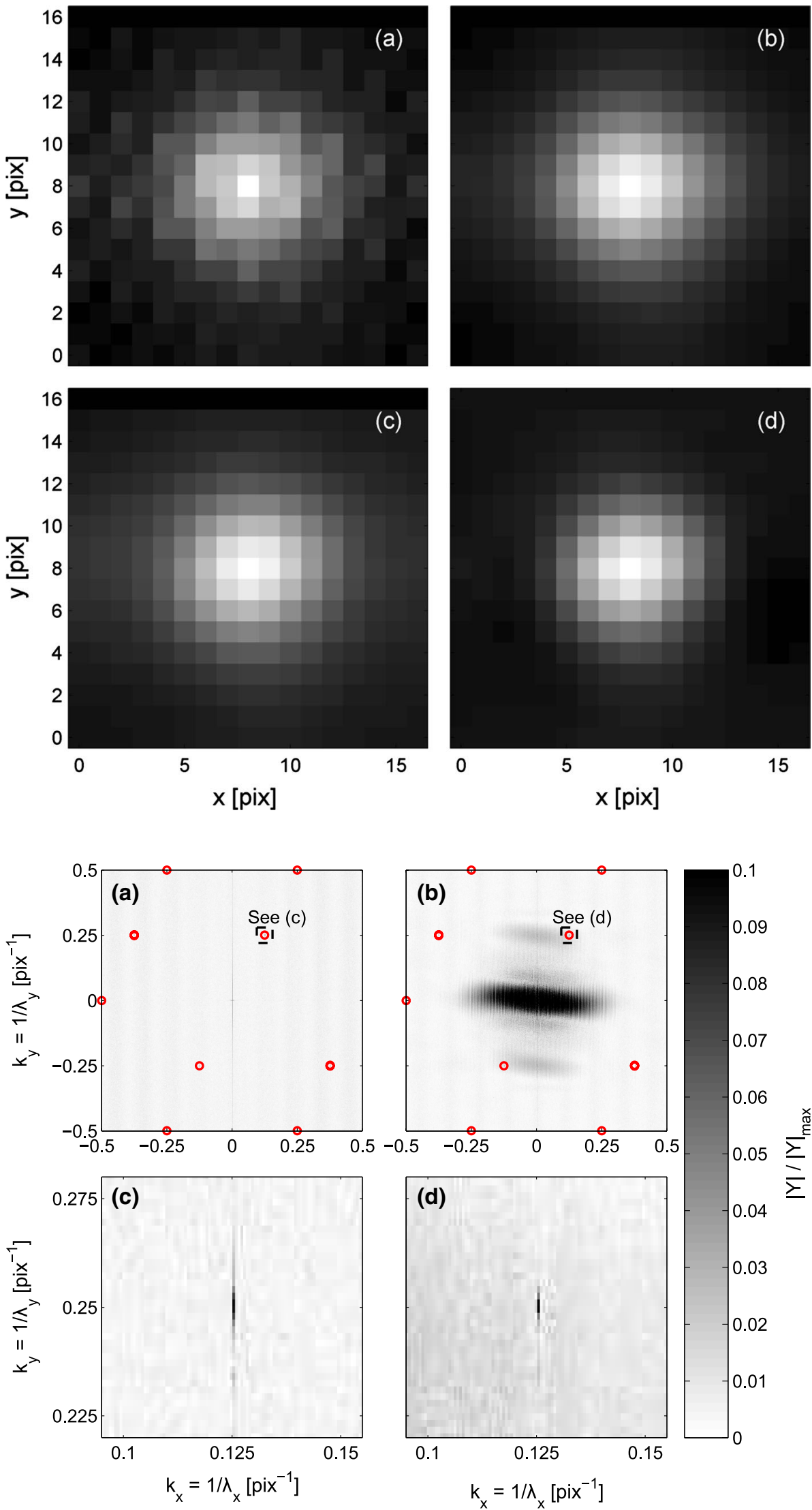
Table 2 Processing settings used for the PIV images

\begin{tabular}{|c|c|c|c|c|}
\hline \multirow[t]{2}{*}{ Configuration } & \multicolumn{2}{|c|}{ Interrogation windows } & \multicolumn{2}{|l|}{ Vector pitch } \\
\hline & pixels & $\mathrm{mm}^{2}$ & $x$-direction ( $\mathrm{mm} /$ vector) & $y$-direction $(\mathrm{mm} /$ vector $)$ \\
\hline Boundary layer study & $256 \times 8$ & $1.97 \times 0.06$ & 0.49 & $7.7 \times 10^{-3}$ \\
\hline Oblique shock wave reflection & $96 \times 8$ & $0.74 \times 0.06$ & 0.19 & $7.7 \times 10^{-3}$ \\
\hline
\end{tabular}

\subsubsection{Correction for plate vibrations}

The plate vibrates slightly during the run $(0.03 \mathrm{~mm}$ RMS); however, due to the high-magnification measurements, this corresponds to wall displacements of $\sim 4$ pixels in the images. As a result, particle reflections from the wall sometimes enter the domain used for calculating the velocity profiles. While conversely, actual particle pairs are sometimes covered by the mask that is applied to mitigate laser reflections. Therefore, a procedure has been implemented that identifies the wall location in every image and then shifts all images to a nominal reference position. The wall identification method takes advantage of the particle reflections that are present in the image and positions the wall at the midpoint between the particles and their reflections.

The determination of the wall location is an iterative process. First, a wall location is assumed, and the particle images below the wall are mirrored and correlated with the particle images above the wall. Ideally, for the correct wall location, the process should return a correlation peak in the origin and a correlation coefficient of 1 . In practice, however, the exact location of the peak cannot be determined accurately enough due to vertical streaks in the reflections, and therefore, only the correlation peak coefficient is used as indicator. So, for a range of possible wall locations, the correlation coefficients are computed, and the location that results in the highest coefficient is identified as the best approximation of the wall location.

\subsubsection{Normalization of particle intensities}

The raw PIV images contain particles of widely varying intensities. To normalize the image contrast, a min-max filter is employed with a kernel size of 6 pixels, which is empirically found to deliver the best results. Min-max filtering reduces the intensity of the large/bright particles and increases the intensity of the smaller and weaker particles. This consequently reduces the average particle image diameter from 3.6 pixels before filtering to 2.4 pixels after filtering. This effect is clearly visible in the correlation peak of Fig. 4d, which is narrower than the one obtained without min-max filtering (Fig. 4c). It is generally conceived that the three-point Gaussian peak estimators work best for particle diameter of 2-3 pixels (Raffel et al. 2007).

\subsection{PIV processing}

Because of the very thin boundary layer $(\sim 0.2 \mathrm{~mm}$ at $40 \mathrm{~mm}$ from the leading edge), only a limited number of pixels are available that can be used to reconstruct the velocity profile. At the given spatial resolution (see Table 1) of 130 pixels $/ \mathrm{mm}$, this results in 26 pixels. So in order to have sufficient measurement points inside the boundary layer, a thin and elongated interrogation window is required. For the boundary layer study, windows of $8 \times 256$ pixels were used, which corresponds to $0.06 \mathrm{~mm}$ in the wall-normal direction and $1.97 \mathrm{~mm}$ in the streamwise direction. A window overlap of $75 \%$ was used in the streamwise direction, and an overlap of $87.5 \%$ was used in the wall-normal direction, resulting in vector pitches of $0.49 \mathrm{~mm}$ and $7.7 \mu \mathrm{m}$, respectively. These settings are summarized in Table 2, together with the settings that were used for processing the data from the SWBLI measurements.

Furthermore, window (Gaussian) weighting is applied in order to further decrease correlation noise (Astarita 2007). The resulting velocity fields are post-processed with a normalized median filter to remove spurious vectors (Westerweel and Scarano 2005), and vector relocation (Theunissen et al. 2008) is performed on vectors for which the interrogation window is overlapping the wall mask.

Because of the thin elongated interrogation windows and the relatively low seeding density close to the wall (see Sect. 4.3), single image pair correlation is not well suited for resolving the laminar/turbulent boundary layer profile. Instead an ensemble correlation approach (Meinhart et al. 2000) is used, which builds up the correlation plane by calculating and summing up the correlation results for all the image pairs. For the boundary layer study, a total of 300 image pairs, and for the measurements on the SWBLI, 600 image pairs were collected per field of view.

The image pre-processing steps were performed in MATLAB, and the ensemble correlation technique was implemented in our in-house-built iterative multi-grid window deformation PIV code Fluere, which is based upon the work of Scarano and Riethmuller (2000).

\subsection{Uncertainty analysis}

The PIV results are subject to uncertainties associated with the ensemble correlation approach, particle slip and variations in the wall location. A short discussion is given in this 
section on how these uncertainties may influence the measured velocity fields.

The ensemble correlation approach (Meinhart et al. 2000) is used to determine the average velocity fields for all cases investigated in this study. The quality of the velocity field improves when more images are included in the data set, but is limited by the finite accuracy of the three-point Gaussian peak-fitting procedure used for locating the correlation peak. An a posteriori approach to determine the corresponding uncertainty is possible by considering the velocity variations in the freestream. In an ideal situation, the converged ensemble-averaged velocity in the freestream should yield the same value, and small spatial variations with respect to this value can be attributed to the finite accuracy of the peak-fitting procedure. The root-mean-square (RMS) value of the velocity variations is found to be $0.096 \%$ of $U_{\infty}$ or equivalently 0.023 pixels for a data set of 600 images. This value forms a lower bound on the uncertainty introduced by the ensemble correlation approach.

In a turbulent boundary layer, it is much harder to build up a correlation peak than in the freestream due to the effects of shear and velocity fluctuations, which both tend to broaden the correlation peak (Scharnowski et al. 2012). By considering the measured velocity variations with respect to the log-law, an approximate upper bound can be given for the ensemble correlation uncertainty. For a fully turbulent boundary layer $(x=100 \mathrm{~mm}$, no impinging shock wave), it is found that the velocity variations with respect to a fitted Van Driest log-law equal $0.73 \%$ of the local $U$, or equivalently 0.12 pixels. The uncertainties introduced by the ensemble correlation approach are therefore expected to fall in between 0.096 and $0.73 \%$ of the local velocity $U$.

The tracer particles that were used for this experiment have a response time of $2.48 \mu \mathrm{s}$, which for the transitional flat-plate boundary layer corresponds to a Stokes number of $\sim 3.7$. This is a rather high value, which according to the paper of Samimy and Lele (1991) may result in slip velocities of up to $25 \%$ of the local velocity. The present investigation will therefore only address the average velocity fields and will not deal with the unsteady effects of the interaction.

In order to calculate the development of the integral parameters throughout the boundary layer, it is important to accurately determine the location of the wall. As discussed in Sect. 3.1, a method has been developed that identifies the wall location in every PIV image and shifts the images to a nominal reference position. The accuracy of this method is conservatively estimated to be 1 pixel. For the laminar boundary layer, this translates into an uncertainty of $6 \%$ on the incompressible displacement thickness $\delta_{i}^{*}, 2.5 \%$ on the incompressible momentum thickness $\theta_{i}$ and $<3.5 \%$ on the incompressible shape factor $H_{i}$. For the fully turbulent boundary layer, the uncertainties related to the wall location are smaller than $2.5 \%$ for the incompressible displacement thickness, $2 \%$ for the momentum thickness and $1 \%$ on the shape factor. The lower uncertainties for the turbulent boundary layer are related to the increased boundary layer thickness, which makes the relative impact of a one-pixel offset on the integral parameters much smaller.

\section{Boundary layer study}

Before a transitional SWBLI can be studied, it is important to first have a good characterization of the undisturbed boundary layer and in particular the transition process. A combination of measurement techniques has been employed for this purpose, namely: oil-flow and spark-light Schlieren visualizations and PIV. The results will also be compared to that of an infrared thermography study (Giepman et al. 2015) performed on a partial-span flat plate in the same wind tunnel under the same conditions.

\subsection{Oil-flow visualization}

The oil-flow visualization (Fig. 6) shows that on average the boundary layer transitions into a turbulent state $\sim 70 \mathrm{~mm}$ from the leading edge of the flat plate. The first streaks are, however, already formed upstream of this location, and the transition onset location appears to be located around $x=55-60 \mathrm{~mm}$. In this particular visualization, two large turbulent wedges are observed that start almost directly behind the leading edge. These local regions of turbulent flow are not caused by plate imperfections, as they appear at different locations for different runs. Instead, they are the result of small accumulations of $\mathrm{TiO}_{2}$ particles that are present in the applied oil film layer. Although these accumulations are strictly spoken unwanted, they do make the distinction between laminar and turbulent flows very clear. Finally, the transition region is nominally two-dimensional over $\pm 85 \mathrm{~mm}$ from the centreline, which corresponds to approximately $62 \%$ of the plate span.

\subsection{Spark-light Schlieren visualizations}

Spark-light Schlieren measurements were performed on the flat-plate boundary layer. The spark-light delivers a short light pulse of $20 \mathrm{~ns}$, thus instantaneously freezing the flow in the Schlieren image. This allows the visualization of the instantaneous turbulent features in the flow and therefore also to distinguish the laminar from the turbulent part of the boundary layer. The transition location is shown (see Fig. 7) to fluctuate between 55 and $65 \mathrm{~mm}$ during the course of a wind tunnel run. Such fluctuations are consistent with the intermittent character of boundary layer transition. Since the Schlieren visualizations present a spanwise-averaged image of the flow, any turbulent portion of the boundary layer 


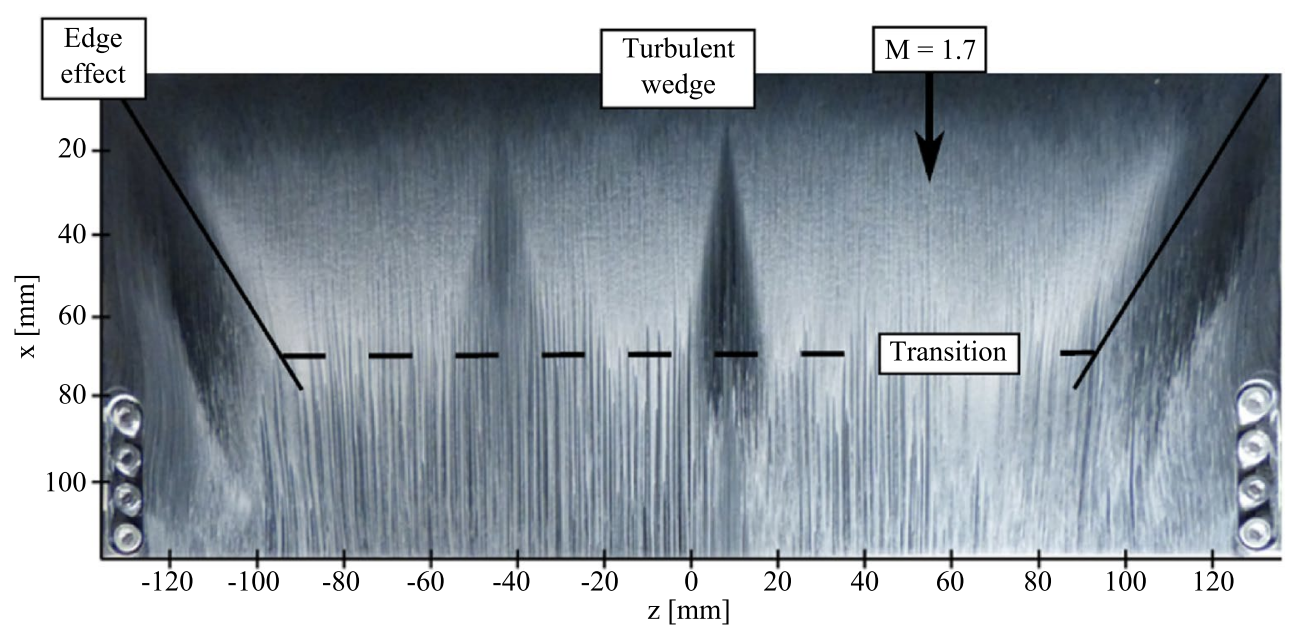

Fig. 6 Oil-flow visualization for the undisturbed boundary layer
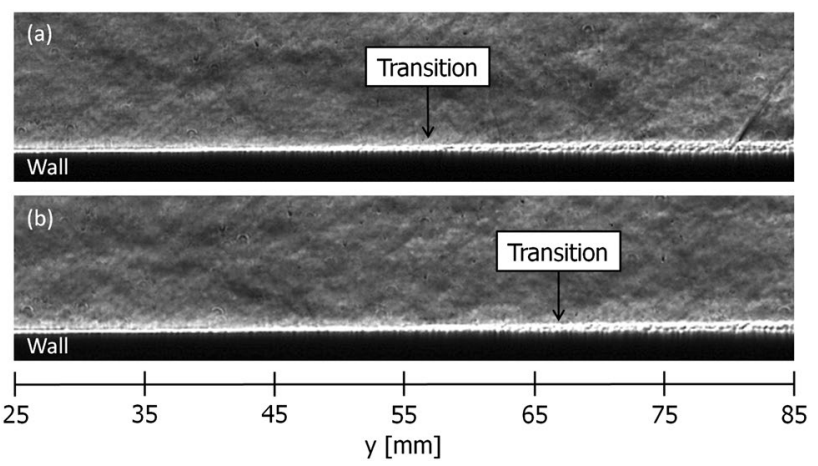

Fig. 7 Spark-light Schlieren visualization of boundary layer transition. Two snapshots are presented to highlight the intermittent character of the transition region

will conceal the laminar portion of the boundary layer. The lower bound $(x=55 \mathrm{~mm})$ that is found from the Schlieren visualizations should therefore be interpreted as the transition onset location of the boundary layer.

\subsection{Particle image velocimetry}

The major challenge of the PIV measurements proved to be the adequate seeding of the laminar boundary layer.
Figure 8 illustrates the average seeding distribution in the flow over the plate and is obtained by stitching together a total of 12 fields of view. From this figure, it is clear that there is a deficiency of seeding in the near-wall region of the flow up to about $80 \mathrm{~mm}$ downstream of the leading edge, which corresponds to the laminar and transitional region of the boundary layer. This behaviour is attributed to the high local streamline curvature near the leading edge (1) and the high level of rotation (large $\mathrm{d} u / \mathrm{d} y$ ) in the newly formed laminar boundary layer (2).

Regarding point (2), a particle entering the boundary layer initially moves faster than the surrounding fluid, and under these conditions, the particles are subjected to a positive lift force, pushing them away from the wall. Auton (1987) showed that (for incompressible/inviscid fluid) the acceleration experienced by the particle is proportional to the velocity gradient $\mathrm{d} u / \mathrm{d} y$ and the slip velocity, the latter being a function of the particle size. Larger particles have a longer response time and will experience larger slip velocities. The boundary layer therefore acts like a filter, with the smallest particles located closest to the wall and the largest particles pushed away from the wall. Since there is no mixing taking place in a laminar boundary layer, there is no mechanism that moves the ejected particles back towards the wall. So, the gap initially present between the wall and

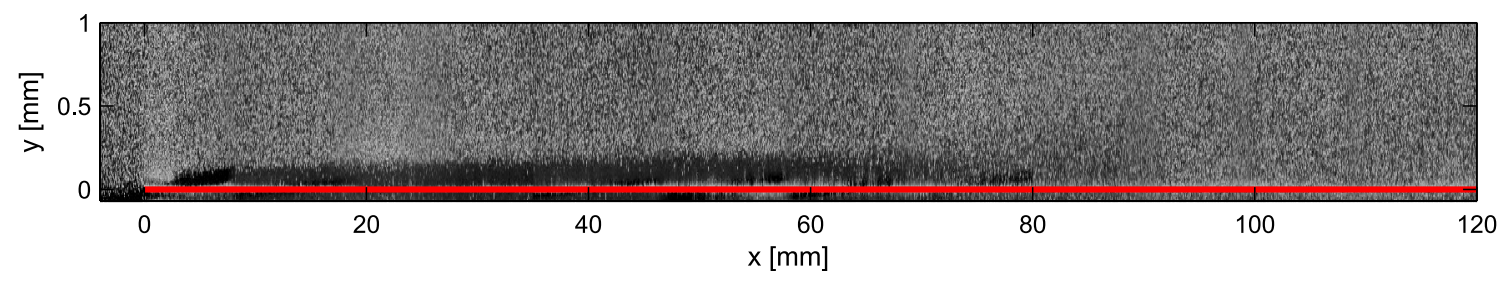

Fig. 8 Average seeding distribution along the plate. The solid red line indicates the wall location 

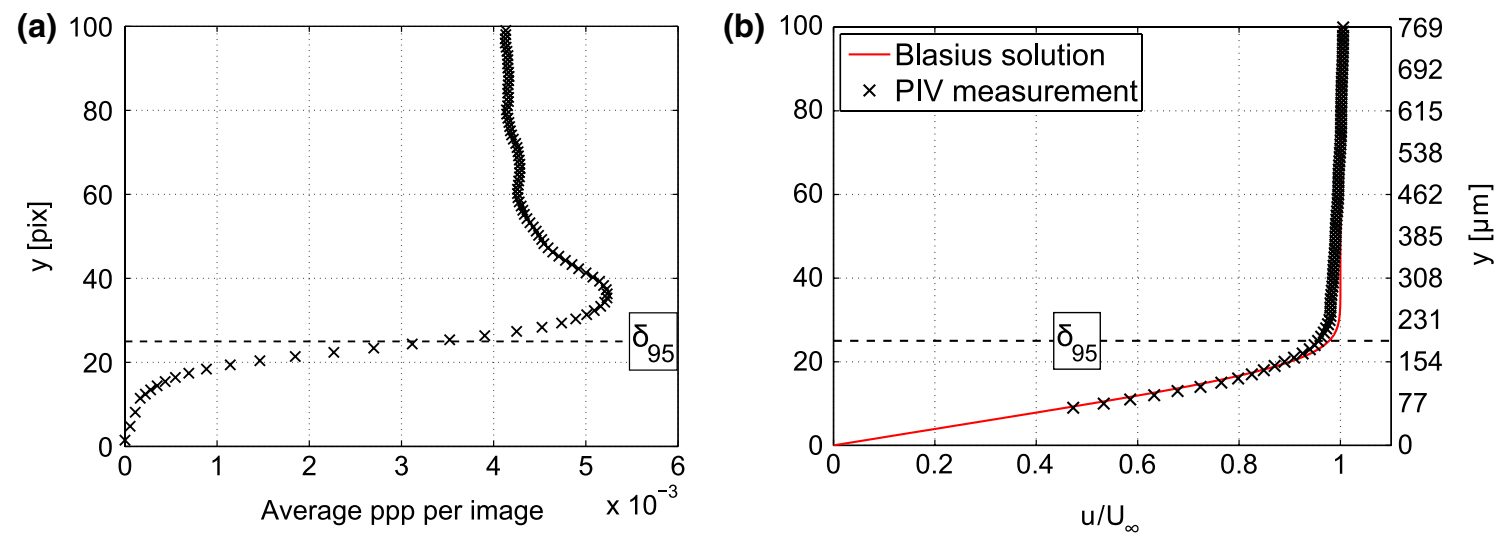

Fig. 9 Average particle distribution in the laminar boundary layer, $40 \mathrm{~mm}$ from the leading edge (a) and the corresponding velocity profile (b)

the properly seeded flow region remains and even grows when moving downstream. The latter is due to the fact that as the boundary layer grows, the streamlines move away from the wall.

A further illustration of the seeding distribution is given in Fig. 9a, which shows the average particles per pixel (ppp) per image at $40 \mathrm{~mm}$ from the leading edge. Virtually no seeding is detected in the first 10 pixels from the wall (which corresponds to $\sim 40 \%$ of $\delta_{95}$ ). Just outside of the boundary layer, at approximately 35 pixels $(0.27 \mathrm{~mm})$ from the wall, an accumulation of particles is found that is the result of the particles having migrated away from the wall. At $40 \mathrm{~mm}$ from the leading edge, $\delta_{95}$ is reached at $\sim 25$ pixels from the wall. The lower 10 pixels provide virtually no information for reconstructing the velocity field due to the lack of seeding, but the remaining 15 pixels can still be used to extract the top $60 \%$ of the boundary layer profile.

The resulting boundary layer profile is presented in Fig. 9b together with the theoretical compressible Blasius solution (obtained by applying the Illingworth (1950) transformation to the incompressible Blasius solution). For $u \leq 0.95 U_{\infty}$, an excellent agreement is obtained between the experimental data and the Blasius solution. Note here that the Blasius solution has not been fitted to the experimental data, but follows directly from theory. For $u \geq 0.95 U_{\infty}$, a small discrepancy can be noted between the experimental data and the Blasius solution. The theoretical Blasius profile quickly reaches its freestream velocity, whereas the experimental data show a longer and smoother transition to the freestream velocity.

So, even though there is virtually no seeding present in the 10 pixels closest to the wall, it is still possible to resolve the remaining $60 \%$ of the boundary layer profile. Since the experimental results agree well with the compressible Blasius solution, it can be used as a basis to extrapolate the experimental data to the wall. In Fig. 8, one can also see that the gap without seeding slowly disappears in the region of $60-80 \mathrm{~mm}$ from the leading edge. These values agree well with the extent of the transition region as determined by means of oil-flow and spark-light Schlieren visualizations. Figure 8 , however, provides an averaged view of the laminar-to-turbulent transition process. When considering individual images, taken at the same downstream position, an intermittent behaviour is observed (see Fig. 10), in which the empty near-wall region is present in some images and absent in others. This confirms that in the transition regime, the boundary layer is switching between a laminar and turbulent state. In the images that show a lack of seeding near the wall, the boundary layer is likely to be laminar, whereas in images showing seeding all the way towards the wall as a result of increased mixing, the flow is most likely turbulent.

To further analyse this intermittent behaviour, the ppp levels were calculated in windows covering the region from 5 to 15 pixels of the wall (256 pixels were used in streamwise direction, $75 \%$ overlap) for every individual image. If the ppp level in the window reached a value higher than $30 \%$ of the freestream ppp level for that particular image, it was qualified as turbulent and otherwise it would be qualified as laminar. The threshold value of $30 \%$ is based upon the fact that upstream of the transition region the intermittency $\gamma$ should approach a value of 0 , whereas downstream of the transition region it should approach a value of 1 . Selecting the threshold value too low/high results in too many samples being classified as turbulent/laminar. The exact level of the threshold has little effect though on the average transition location. For threshold values in the range of $15-45 \%$ a variation of $\pm 2 \mathrm{~mm}$ is noted on the estimated average transition location.

Figure 11 shows the results of the intermittency analysis and although there is some scatter present in the experimental data, one can clearly discern a smooth transition from laminar to turbulent flow taking place over approximately $35 \mathrm{~mm}$. The distribution shows an inflection point 

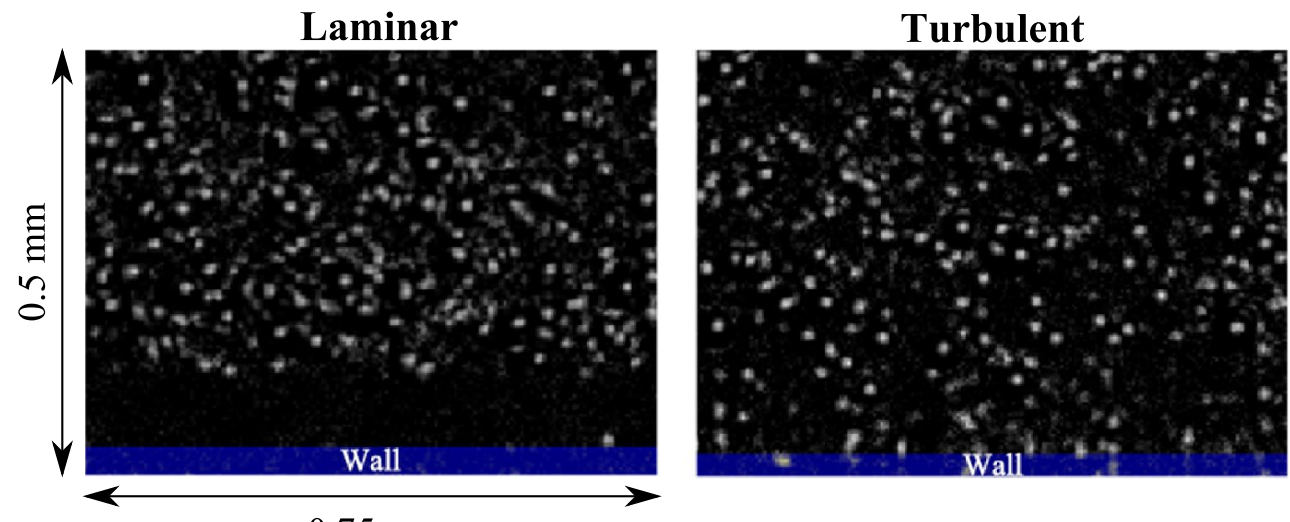

$0.75 \mathrm{~mm}$

Fig. 10 Comparison between laminar/turbulent seeding conditions in the near-wall region of the flow. The field of view is centred around $x=70 \mathrm{~mm}$ from the leading edge

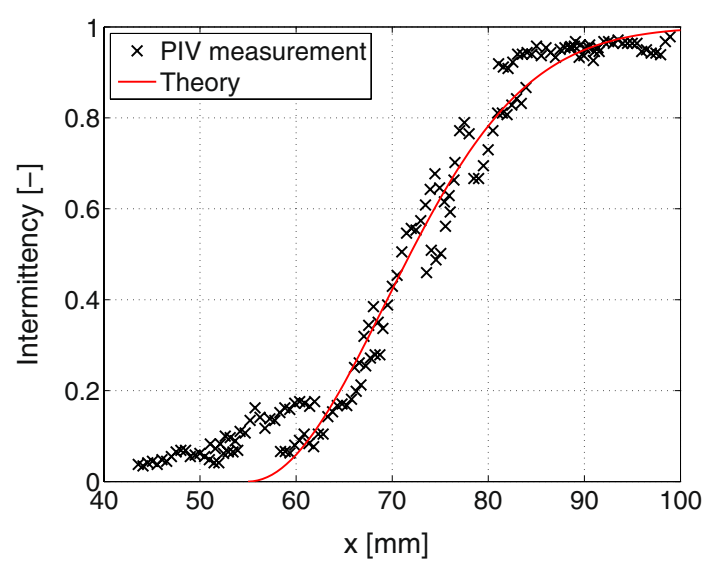

Fig. 11 Intermittency distribution throughout the interaction region

around $x=71 \mathrm{~mm}$, where the boundary layer is laminar half of the time and turbulent the other half of the time.

The experimental data are compared to the semi-empirical intermittency distribution of Dhawan and Narasimha (1958), which is given by the following relation:

$\gamma=1-e^{-0.412 \xi^{2}}$

with $\gamma$ being the intermittency and $\xi=\left(x-x_{\mathrm{T}}\right) / \lambda$. Here $x_{\mathrm{T}}$ is the onset location of the transition region and $\lambda=x_{\gamma=0.75}-x_{\gamma=0.25}$, which denotes the distance between the $x$-locations where the flow is turbulent 75 and $25 \%$ of the time. Based upon the experimental data from Fig. 11, $x_{\mathrm{T}}=55 \mathrm{~mm}$ and $\lambda=13 \mathrm{~mm}$. Equation (2) is based on the hypothesis of a localized laminar breakdown and the formation of turbulent spots in a very small restricted region around $x_{\mathrm{T}}$, an assumption valid for most 2D laminar flatplate boundary layers (Narasimha 1957).

Both the experimental data and the model of Dhawan and Narasimha (1958) are subject to a certain degree of empiricism and some discrepancies may be noted near the onset and the end of the transitional regime. The theoretical model predicts that the intermittency approaches zero at $x_{\mathrm{T}}=55 \mathrm{~mm}$, whereas the experimental data appear to level off at an intermittency level of $\gamma \approx 0.04$. This is due to the fact that in some of the images wall reflections are entering the search domain ( $y=5-15$ pixels) and are classified as real particles, resulting in a nonzero intermittency level. Notwithstanding these differences, it appears that the experimental data and the model of Dhawan and Narasimha (1958) both deliver the same general trend, which indicates that the seeding density in the near-wall region is a suitable indicator for the state of the boundary layer.

This knowledge can be further exploited to improve the quality of the transitional velocity profiles as obtained by cross correlation. In the laminar and turbulent regime, the velocity vectors can be determined in a relatively straightforward manner using the ensemble correlation technique. However, in the transition region, the flow is switching between two distinct flow states, which makes it hard to construct a clean ensemble-averaged correlation peak and consequently makes the computation of the location of this peak unreliable. This has motivated to adopt a data-processing procedure in which the image pairs are divided into two sets, of assumed laminar and turbulent flow images, which have a low and high near-wall seeding density, respectively. The ppp level in the region of 5-15 pixels from the wall is used as the indicator for the laminar/turbulent character of the boundary layer (following the same procedure as used for Fig. 11 of the intermittency distribution). On both data sets, the ensemble correlation technique is applied separately, delivering two velocity fields. For the velocity field corresponding to the laminar subset, no velocity vectors can be calculated in the near-wall region of the flow ( $\leq 10-15$ pixels). The Blasius solution is therefore used to extrapolate the vectors outside of this region down to the 


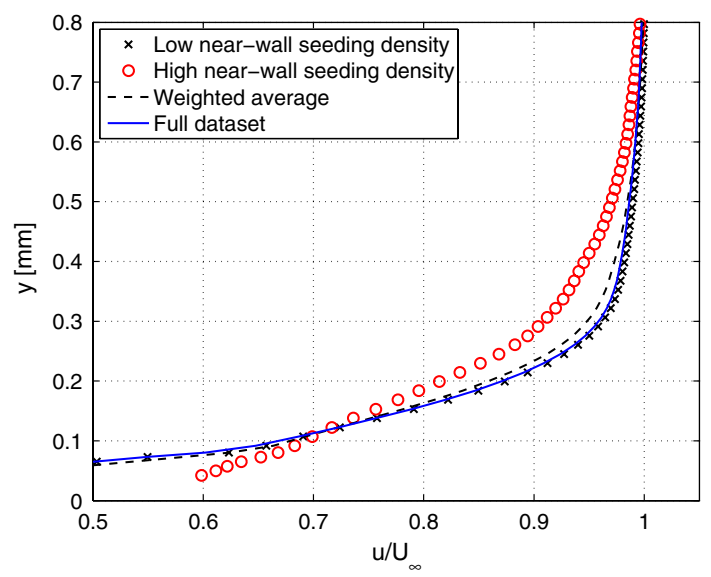

Fig. 12 Intermittency-based weighting of a transitional velocity profile $(x=66 \mathrm{~mm}, \gamma=0.25)$

wall. For the velocity field corresponding to the turbulent subset, all vectors are used. A weighted average (based on the intermittency level) of the two velocity fields is subsequently computed to obtain the velocity field in the transition region.

Figure 12 illustrates this weighting procedure on a velocity profile located at $x=66 \mathrm{~mm}$, where the flow has an intermittency level of approximately $25 \%$. As expected, the subset with the low near-wall seeding density shows an almost laminar profile $\left(H_{i}=2.00\right)$, and the subset with high near-wall seeding density shows a turbulent velocity profile $\left(H_{i}=1.40\right)$. The velocity profile that is obtained by processing the full data set is very close $\left(H_{i}=1.95\right)$ to the profile that is obtained by processing the images with a low nearwall seeding density. That is because the flow at $x=66 \mathrm{~mm}$ is still mostly laminar, and consequently in the ensemble correlation plane, the strongest peak will be formed for the laminar option and not for the turbulent option. The resulting velocity profile is therefore biased towards the laminar solution if no correction is applied. By dividing the data set into subsets of low- and high-seeding density and by applying the proper weighting, a better approximation can be obtained for the transitional velocity profile. The incompressible shape factor for the weighted solution is $H_{i}=1.85$ in this case. The same behaviour is also found for intermittency values larger than $50 \%$, but now the bias is towards the turbulent solution instead of the laminar one.

The development of the boundary layer profile, from laminar, transitional to turbulent, is illustrated in the six frames of Fig. 13. At $x=50$ and $60 \mathrm{~mm}$, the data points are in good correspondence with the compressible Blasius solution. In the region from 70 to $90 \mathrm{~mm}$, the data points start to deviate from the laminar profile and approach the theoretical turbulent profile. At $100 \mathrm{~mm}$ from the leading edge, the boundary layer has reached a fully turbulent state.
Figure 14 shows a semi-logarithmic representation of the latter turbulent profile $(x=100 \mathrm{~mm})$ in terms of the inner variables $y^{+}=y u_{\tau} / v_{w}$ and $u^{+}=u_{\mathrm{eq}} / u_{\tau}$. Here $u_{\tau}=19.6 \mathrm{~m} / \mathrm{s}$ presents the friction velocity, $\nu_{\mathrm{w}}$ is the kinematic viscosity evaluated at the wall, and $u_{\mathrm{eq}}$ is the Van Driest effective velocity (Van Driest 1951). The log region can be clearly distinguished in the PIV data, and also the start of the viscous sublayer is resolved. Figure 14 also highlights the need for vector relocation (Theunissen et al. 2008) when performing accurate near-wall measurements. Without relocation, the near-wall velocity vectors are placed too close to the wall, and the resulting velocity is biased towards a higher value. This trend is clearly visible in Fig. 14, which shows that without vector relocation, a reliable estimate of the velocity can be given down to $y^{+}$of 27 , and with vector relocation, it is possible to reach a $y^{+}$of 19 . In physical units, this translates into 38 and $27 \mu \mathrm{m}$, respectively (or alternatively 5 pixels and 3.5 pixels from the wall).

Finally, the development of the incompressible displacement thickness $\delta_{i}^{*}$, incompressible momentum thickness $\theta_{i}$ and the incompressible shape factor $H_{i}$ is displayed in Fig. 15. The shape factor starts at a value of approximately 2.9 at $40 \mathrm{~mm}$ from the leading edge (the Blasius solution predicts a slightly lower shape factor of 2.7 under these conditions) and from 55 to $85 \mathrm{~mm}$ experiences a gradual drop to a value of $\sim 1.35$, typical for a turbulent boundary layer. The momentum thickness shows a monotonic increase along the flat plate, whereas the displacement thickness experiences a clear drop within the transition region. The displacement thickness reaches a local maximum around $x=64 \mathrm{~mm}$, decreases in value until $x=77 \mathrm{~mm}$ and then starts to rise again.

\subsection{Comparison of techniques}

The transition region has been characterized by means of three different techniques: oil-flow visualization, sparklight Schlieren visualization and PIV. Additionally, infrared thermography measurements were carried out in an earlier study (Giepman et al. 2015) on a partial-span (64\%) flatplate model in the same wind tunnel under the same operating conditions. The transition locations that were found by means of the four experimental techniques are summarized in Table 3.

The transition onset location $x_{\mathrm{T}}$ is found to be $55 \mathrm{~mm}$ $\left(R e_{x_{T}}=1.9 \times 10^{6}\right)$ by both spark-light Schlieren visualization and the PIV measurements. The average transition location $x_{\gamma=0.5}$ (intermittency of $50 \%$ ) has been determined by three techniques (oil-flow visualization, infrared thermography and PIV) and equals approximately $\sim 70 \mathrm{~mm}\left(R e_{x_{\gamma=0.5}}=2.5 \times 10^{6}\right)$. The transition length $L$ was derived from the PIV measurements and equals $35 \mathrm{~mm}$. This implies that the process of transition is approximately 


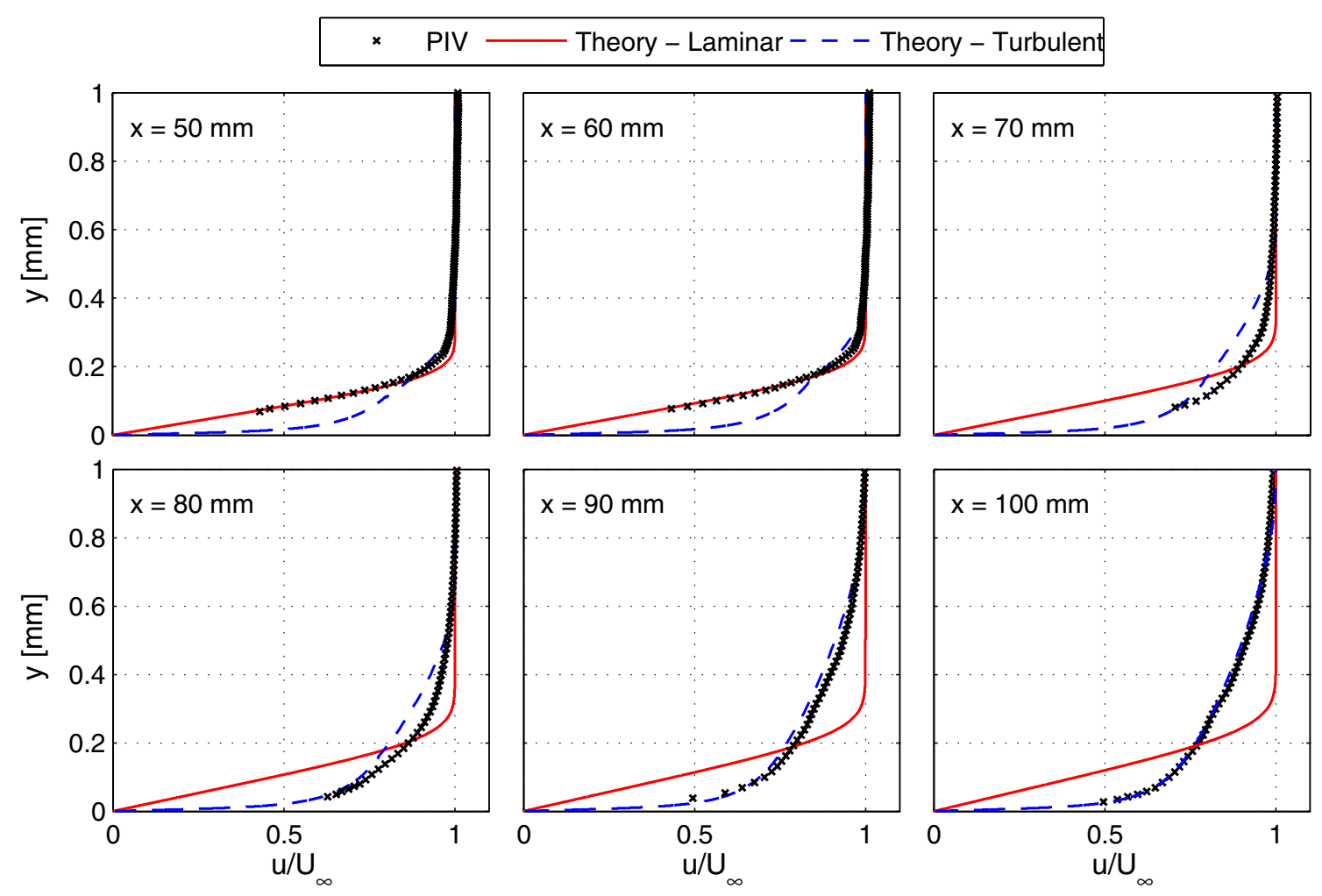

Fig. 13 Development of the boundary layer profile along the plate

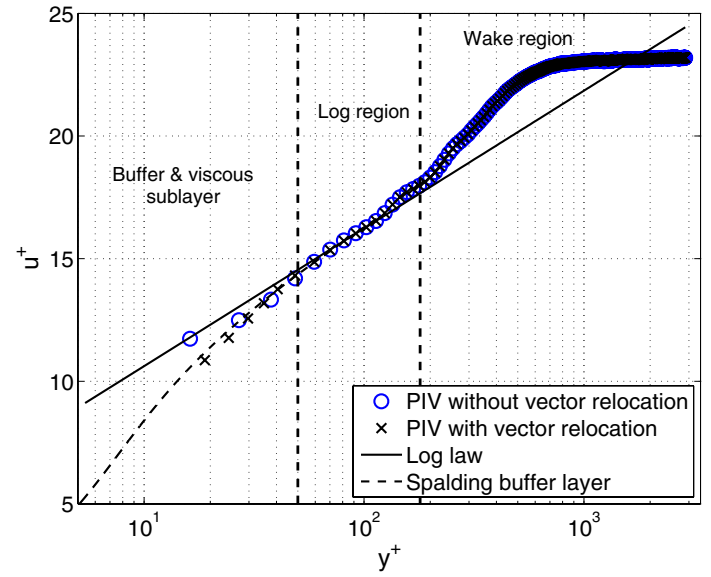

Fig. 14 Undisturbed turbulent boundary layer profile measured at $x=100 \mathrm{~mm}$

restricted to the region of $x=55-90 \mathrm{~mm}$ on the flat plate, or in terms of Reynolds numbers from 1.9 to $3.2 \times 10^{6}$.

\section{Oblique shock wave reflection}

An oblique shock wave was positioned to impinge on the laminar $\left(x_{\mathrm{sh}}=51 \mathrm{~mm}\right)$, transitional $\left(x_{\mathrm{sh}}=71 \mathrm{~mm}\right)$ or turbulent $\left(x_{\mathrm{sh}}=101 \mathrm{~mm}\right)$ region of the boundary layer. For the transitional case, the boundary layer has an intermittency level of approximately $50 \%$ at the shock impingement location (see Fig. 11). The shock generator was set to induce a flow deflection angle of $3^{\circ}$, which corresponds to an overall theoretical pressure ratio of 1.35 (based on an inviscid shock reflection). For this particular shock strength, it is expected that the laminar SWBLI shows a significant portion of reversed flow, whereas the turbulent SWBLI remains attached (see Sect. 2.2). The three interactions were investigated by means of oil-flow visualizations, high-speed Schlieren visualizations and PIV.

\subsection{Oil-flow visualizations}

Oil-flow visualizations were performed to investigate the wall flow topology for the laminar, transitional and turbulent interactions. The images shown in Fig. 16 were acquired while running the tunnel, because it was observed that shutting down the tunnel has a significant effect on the oil film pattern (in particular the separated regions). For the laminar case (Fig. 16a), a large separation bubble $(\sim 10 \mathrm{~mm}$ in length) is formed. The size of the bubble is significantly reduced for the transitional case, and for the turbulent case, not more than a vague white line can be distinguished at the shock impingement location. The thin white line is not 

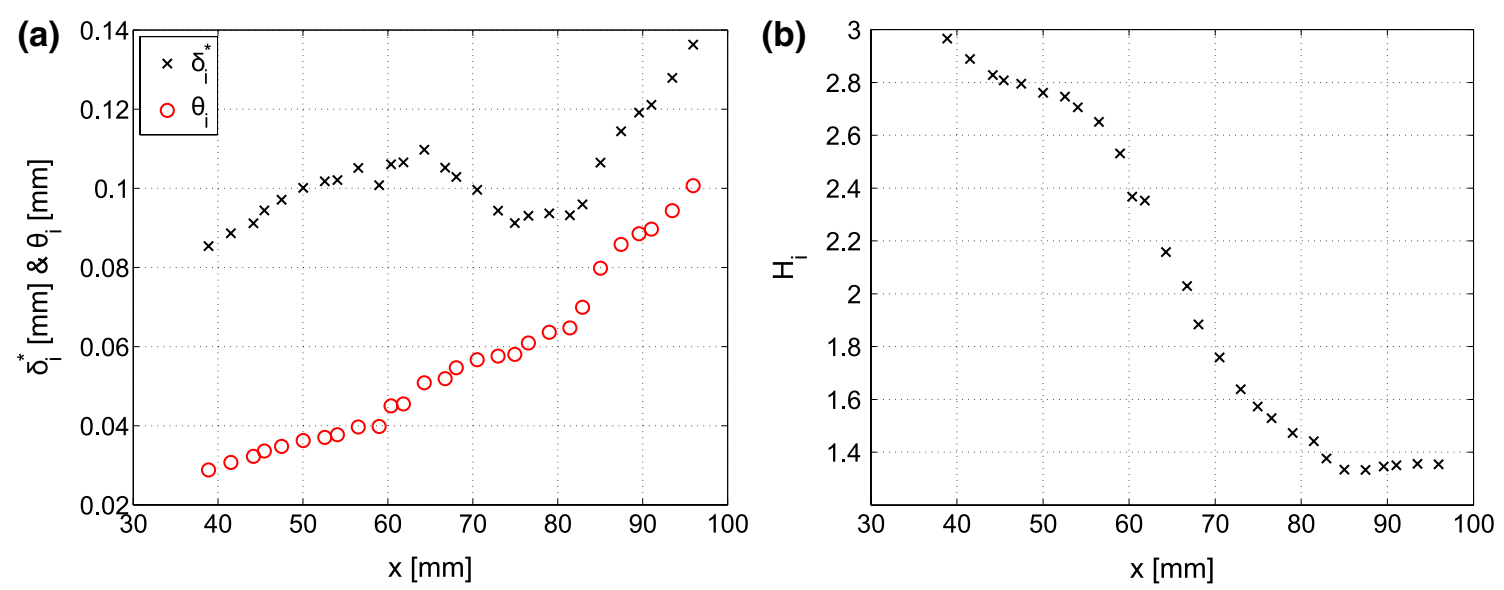

Fig. 15 Development of the integral parameters for the undisturbed boundary layer. Incompressible momentum $\theta_{i}$ and displacement thickness $\delta_{i}^{*}$ (a) and incompressible shape factor $H_{i}(\mathbf{b})$

Table 3 Comparison of the transition locations found with the four different techniques

\begin{tabular}{llll}
\hline & $x_{\mathrm{T}}(\mathrm{mm})$ & $x_{\gamma=0.5}(\mathrm{~mm})$ & $L(\mathrm{~mm})$ \\
\hline Spark-light Schlieren & 55 & & \\
Oil-flow visualizations & $55-60$ & 70 & \\
Infrared thermography & & 68 & 35 \\
Particle image velocimetry & 55 & 71 & \\
\hline
\end{tabular}

due to boundary layer separation, but is associated with the change in skin friction coefficient when crossing the interaction.

\subsection{Schlieren visualizations}

High-speed $(60-\mathrm{kHz})$ Schlieren visualizations provided further information on the flow field of the laminar, transitional and turbulent interactions. No large-scale unsteadiness was found for any of the three cases, and the oscillation amplitude of the reflected shock/reflected compression waves were found to be $<0.3 \mathrm{~mm}$ for all three cases.
The laminar interaction (see Fig. 17a) shows the presence of two compression waves and an expansion fan, a structure which is very similar to what has been reported by other researchers (Gadd et al. 1954; Hakkinen et al. 1959) and which is indicative of a separated interaction. The adverse pressure gradient imposed by the incident shock wave causes the incoming laminar boundary layer to separate well upstream of the shock impingement location, which displaces the freestream. The boundary layer therefore effectively acts as a compression ramp, creating a first series of compression waves. The separation bubble reaches its maximum height around the impingement location of the incident shock (see also the PIV results of Sect. 5.3). From the top of the separation bubble, an expansion fan emanates, which appears as a white region in the Schlieren visualizations. Moving downstream, the boundary reattaches again, and this is accompanied by a series of reattachment waves, also clearly visible in Fig. 17a.

The Schlieren visualization for the transitional interaction of Fig. $17 \mathrm{~b}$ shows a similar structure. For this case, however, the compression waves are confined to a narrower region compared with the laminar interaction. This
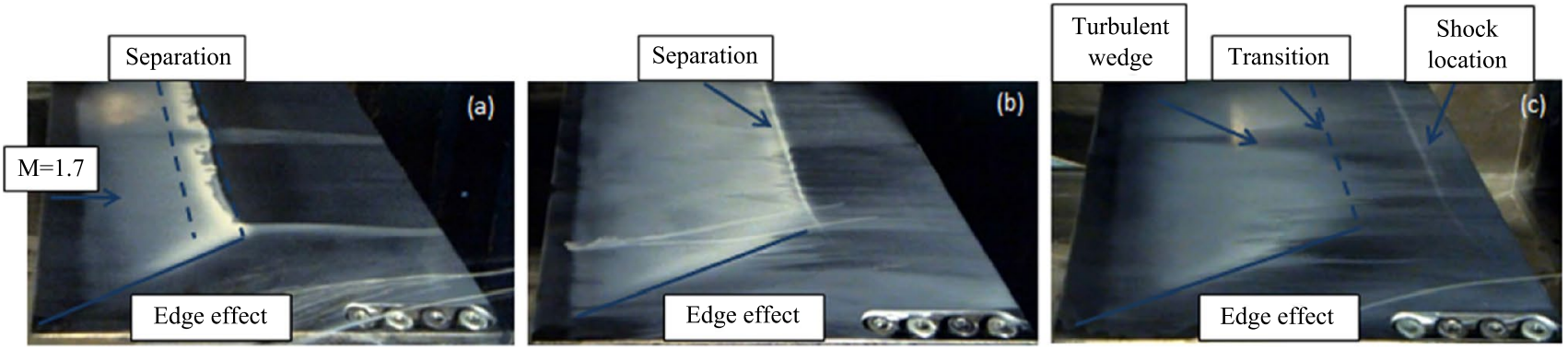

Fig. 16 Oil-flow visualizations for the interaction between an oblique shock wave and a laminar (a), transitional (b) and turbulent (c) incoming boundary layer 


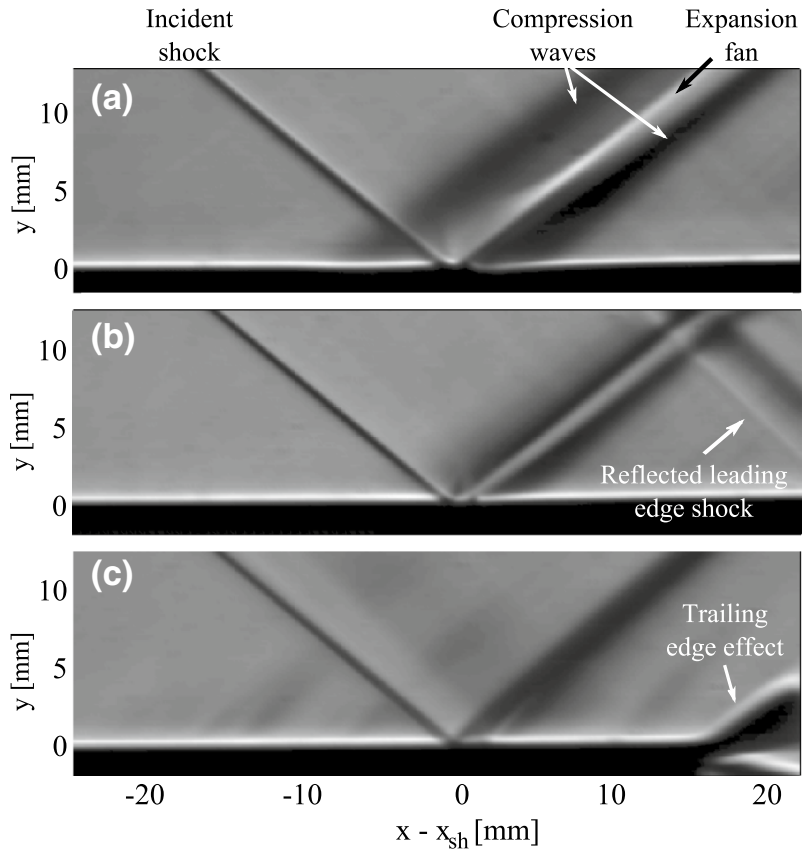

Fig. 17 Schlieren visualizations (time-averaged) for the interaction between an oblique shock wave $\left(\theta=3^{\circ}\right)$ and a laminar (a), transitional (b) and turbulent (c) incoming boundary layer

indicates that the boundary layer is probably still separated, but that the extent of the separated zone is substantially smaller. This conclusion is in line with the oil-flow visualizations reported in the previous section.

Finally, Fig. 17c presents the Schlieren visualization for the fully turbulent interaction. An expansion fan is no longer (clearly) visible, and the incident shock wave is reflected as a series of compression waves confined within a small (2- to 3-mm) region in space. This behaviour is typical for a SWBLI without separation. The 'wave' that is present in the Schlieren image around $x-x_{\mathrm{sh}}=16 \mathrm{~mm}$ is a result of the plate trailing edge, but is sufficiently far downstream to not influence the SWBLI.

\subsection{Particle image velocimetry}

The results of the PIV measurements are presented in Figs. 18 and 19 for the $u$ - and $v$-component of the mean velocity field, respectively. The results are in good agreement with the Schlieren visualizations, and the same flow features can be identified.

For the laminar case (Figs. 18a, 19a), a large separation bubble is present which starts $7.5 \mathrm{~mm}$ upstream of the incident shock impingement location and ends $1.4 \mathrm{~mm}$ downstream of the impingement location. The total length of the bubble therefore measures $8.9 \mathrm{~mm}$, which corresponds to approximately 96 times the undisturbed incompressible displacement thickness $\delta_{i, 0}^{*}$ recorded upstream of the interaction (see Fig. 15). According to the velocity profiles, the incoming laminar boundary layer is lifted over the separation bubble and remains in a laminar state up to the impingement location of the incident shock (see also Fig. 23b). Hereafter, transition takes place, and the boundary layer quickly reattaches at the wall, closing the separation bubble. The incident shock is reflected from the top of the separation bubble as an expansion fan, and compression waves are formed both at the separation and at the reattachment location of the boundary layer.

The transitional case (Figs. 18b, 19b) shows a significantly smaller region of reversed flow $\left(4.3 \mathrm{~mm} ; 43 \delta_{i, 0}^{*}\right)$, which extends $3.9 \mathrm{~mm}$ upstream of the incident shock and $0.4 \mathrm{~mm}$ downstream. From the mean velocity field, two regions of compression waves can be distinguished: one is formed at the separation location of the boundary layer and the other at the reattachment location. A clear expansion wave, as was found for the laminar interaction, is no longer visible in the velocity field.

For the turbulent case (Figs. 18c, 19c), no separation is detected in the average flow field, which is in line with the results obtained by Schlieren and oil-flow visualizations. The interaction length for the turbulent case equals approximately $1.6 \mathrm{~mm}\left(\sim 11 \delta_{i, 0}^{*}\right)$ and is calculated by extrapolating the traces of the incident and reflected shock wave down to the wall.

The seeding difficulties that were encountered when investigating the undisturbed boundary layer (Sect. 4.3) were also present when investigating the laminar and transitional shock wave boundary layer interactions. Although the situation is slightly alleviated by the presence of a separation bubble, which recirculates a small portion of the particles, the seeding level still remains very low compared with the freestream. Figure 20a presents the seeding distribution for the laminar SWBLI. The boundary layer is lifted over the reversed flow region, and due to the initially laminar character of the interaction, the same applies for the seeding. The seeding density is therefore very low in the separation bubble, and the signal is dominated by wall reflections. Consequently, no reliable velocity vectors could be measured in this region of the flow, and the data have been blanked in Figs. 18 and 19.

Although the velocity field in the separation bubble cannot be reconstructed, the data further away from the wall $\left(u>0.2 U_{\infty}\right)$ can still be considered as reliable, because of the higher seeding density and smaller probability of encountering wall reflections. In the work of Lees and Reeves (1964), it was shown that the velocity profiles in the interaction region of a laminar SWBLI may be approximated by Falkner-Skan velocity profiles. It was therefore decided to create a database of Falkner-Skan velocity profiles, containing both separated (lower branch) and attached (upper branch) solutions. These profiles were fitted (see 
Fig. 18 Average velocity field ( $u$-component) of an oblique shock wave reflection $\left(\theta=3^{\circ}\right)$ with an incoming laminar (a), transitional $(\gamma=0.5)(\mathbf{b})$ and turbulent (c) boundary layer. The corresponding shock locations are, respectively, $x_{\mathrm{sh}}=51$, 71 and $101 \mathrm{~mm}$

Fig. 19 Average velocity field ( $v$-component) of an oblique shock wave reflection $\left(\theta=3^{\circ}\right)$ with an incoming laminar (a), transitional $(\gamma=0.5)(\mathbf{b})$ and turbulent (c) boundary layer


Fig. 20b) to the experimental data in the velocity range from 0.2 to $0.6 U_{\infty}$ and used to approximate the height of the reversed flow region ( $u=0$ isoline).

The reversed flow regions for the laminar and transitional interaction are presented in Fig. 21. For the laminar interaction, a maximum height of $0.21 \mathrm{~mm}\left(2.3 \delta_{i, 0}^{*}\right)$ is reached approximately $0.7 \mathrm{~mm}\left(7.6 \delta_{i, 0}^{*}\right)$ upstream of the shock impingement location $x_{\text {sh }}$, which is defined by linearly extrapolating the incident shock wave to the wall.
In practice, the shock will not reflect at the wall, but at the sonic line. Also, the shock will become steeper when entering the boundary layer. These features explain why the maximum height of the reversed flow region is reached slightly upstream of the wall shock impingement location $x_{s h}$. It is furthermore interesting to notice that the reversed flow region has the typical triangular shape expected for a laminar SWBLI (Gadd et al. 1954; Hakkinen et al. 1959). The flow deflection angle of $\alpha=1.8^{\circ}$ for the upstream 

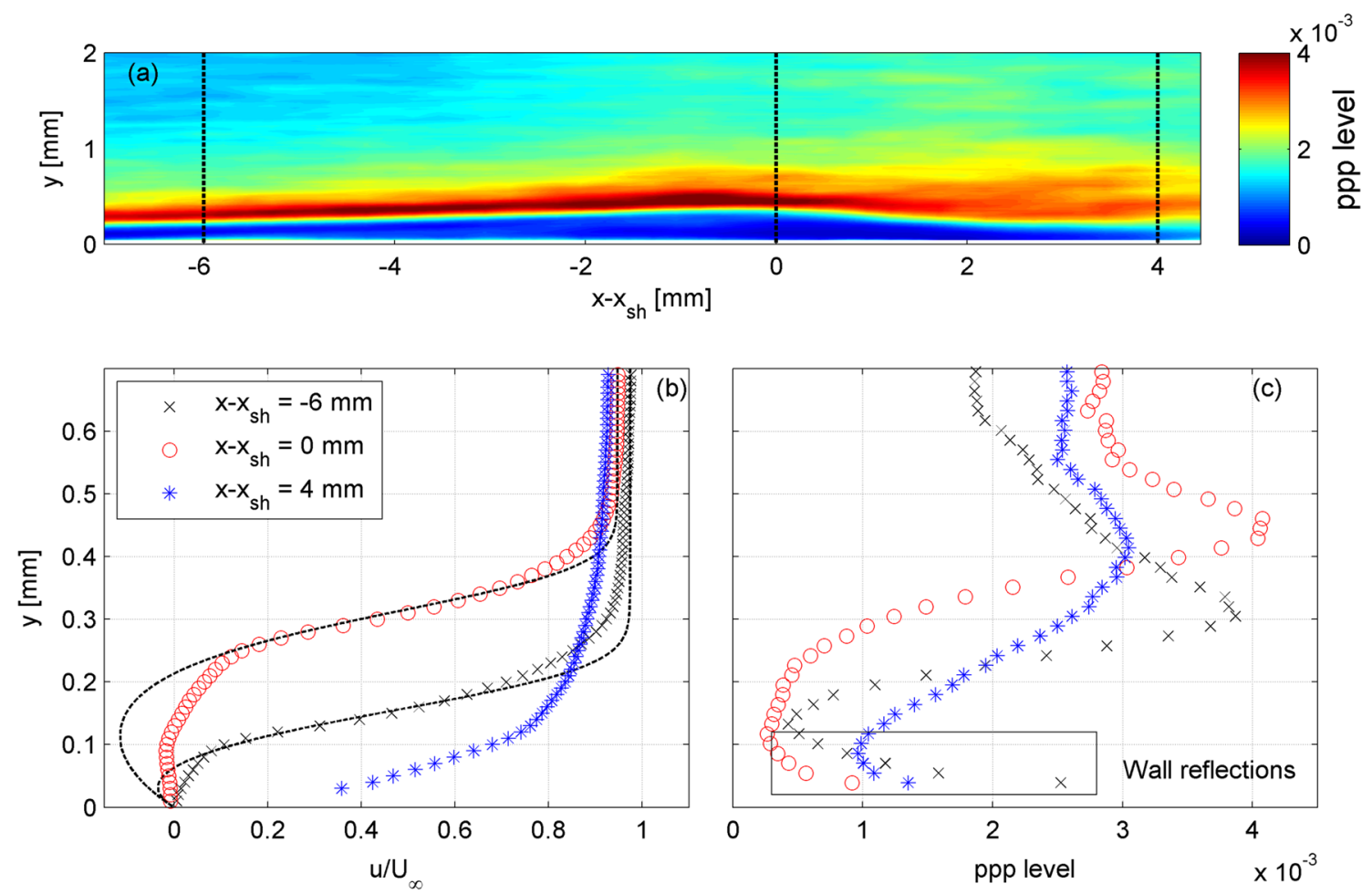

Fig. 20 Analysis of the seeding distribution and velocity profiles for an oblique shock wave reflection with laminar incoming boundary layer $\left(x_{\mathrm{sh}}=51 \mathrm{~mm}\right)$. a Seeding distribution, b velocity profiles; dashed lines represent fitted Falkner-Skan profiles, $\mathbf{c}$ seeding level profiles

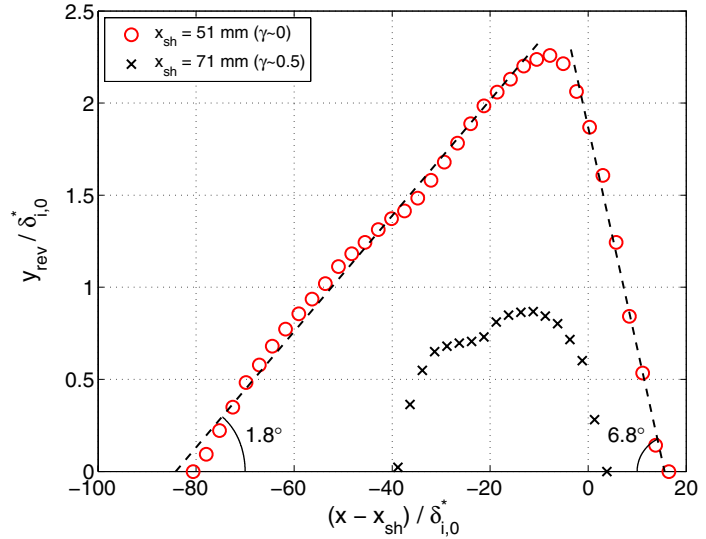

Fig. 21 Reversed flow region for an interaction with a laminar $\left(x_{\mathrm{sh}}=51 \mathrm{~mm}\right)$ and transitional $\left(x_{\mathrm{sh}}=71 \mathrm{~mm}\right)$ incoming boundary layer

portion of the reversed flow region agrees very well with the theory of Hakkinen et al. (1959). They found that $\alpha=\operatorname{atan}\left(0.5 \beta C_{p_{\mathrm{pl}}}\right)$, with $\beta=\sqrt{M^{2}-1}$ and $C_{p_{\mathrm{pl}}}$ the plateau pressure coefficient, for which they have found the following empirical relation: $C_{p_{\mathrm{pl}}}=1.65 \sqrt{2 C_{f_{0}} / \beta}$. The skin friction coefficient upstream of the interaction $C_{f_{0}}$ has been derived from the PIV data and equals $4.8 \times 10^{-4}$. Substituting this value into the relations derived by Hakkinen et al. yields an angle $\alpha=1.7^{\circ}$, which is close to the value of $1.8^{\circ}$ determined from Fig. 21. The angle of the downstream portion of the reversed flow region is much larger $\left(6.8^{\circ}\right)$, which is at least partially caused by the fact that the boundary layer starts to transition when crossing the incident shock wave.

The transitional case shows a much smaller reversed flow region of $43 \delta_{i, 0}^{*}$ in the streamwise direction, and the maximum reversed flow height $\left(0.87 \delta_{i, 0}^{*}\right)$ is achieved approximately $11 \delta_{i, 0}^{*}$ upstream of the shock impingement location. Also, the reversed flow region is no longer triangular, in contrast to the laminar interaction case.

The PIV data that have been gathered during the experiments can be used to track the state (laminar/transitional/ turbulent) of the boundary layer as it develops over the separation bubble. For this purpose, an adapted definition of the incompressible shape factor is introduced. In the classical definition of the shape factor, the integration is performed on the velocity field from the wall up to the freestream. This implies that the separation bubble will have a major effect on the value of the shape factor, which is undesirable if it is to be used as a metric to indicate transition. Therefore, to eliminate the contribution of the separation bubble to the shape factor, a new 'artificial' wall location $y_{\text {aw }}$ is defined by linearly extrapolating the velocity vectors in the range of $0.2-0.6 U_{\infty}$ to $0 \mathrm{~m} / \mathrm{s}$. This implies 
that the data points below $y_{\text {aw }}$ are therefore no longer used when calculating the shape factor. This procedure is illustrated in Fig. 22 for a velocity profile extracted from the laminar SWBLI case at the reattachment location.

Figure 23a shows the shape factor $H_{i}$ as calculated by the classical definition (i.e. $y_{\text {wall }}=0$ ), and Fig. 23b shows the shape factor $H_{i, \text { aw }}$ according to the new definition (i.e. $y_{\text {wall }}=y_{\text {aw }}$ ). Please keep in mind here that no velocity field data are available inside the separation bubble due to the lack of seeding. A Falkner-Skan type of extrapolation was therefore performed (see Fig. 20b) to obtain an approximate velocity field in the separation bubble. Consequently, Fig. 23a only presents a rough approximation of the development of the true shape factor. It is, however, clear from the figure that in this form, the shape factor is not suitable

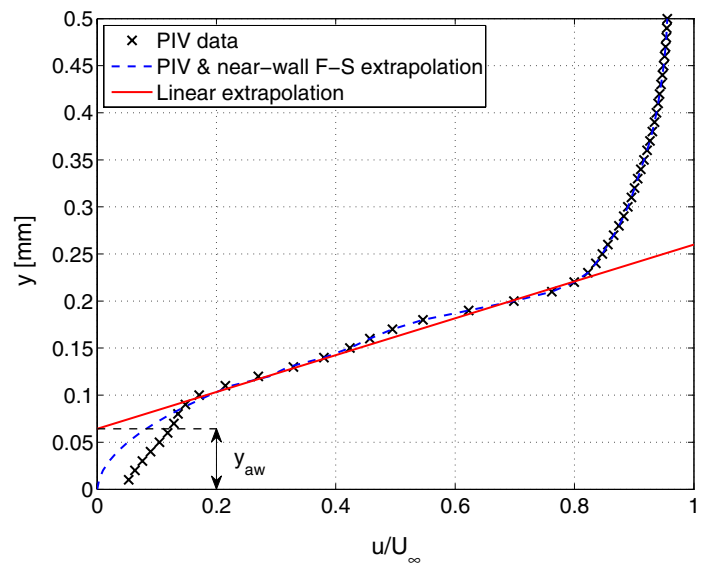

Fig. 22 Procedure for determining a new 'artificial' wall location $y_{\text {aw. }}$. The velocity profile has been extracted from the laminar SWBLI case at the reattachment location $\left(x-x_{\mathrm{sh}}=16 \delta_{i, 0}^{*}\right)$ as a transition indicator, as its development is dominated by the peak that results from the separated flow region.

Figure $23 \mathrm{~b}$ on the other hand provides more insight into the transition behaviour of the boundary layer. For the laminar interaction, it is clear that the boundary layer on top of the separation bubble remains in a laminar state up to the impingement location of the incident shock. After this point, transition sets in and a turbulent boundary layer flow is reached within $80-90 \delta_{i, 0}^{*}$, which is approximately $60-70 \delta_{i, 0}^{*}$ downstream of the reattachment location of the boundary layer. For the transitional interaction, it can be noticed that the boundary layer shows a strong development over the separation bubble. Starting from a nearly laminar boundary layer, it develops into a turbulent profile before the point of reattachment. At reattachment, the boundary layer profile temporarily becomes slightly less full (with a rise in the shape factor), but a quick recovery is observed, and within $\sim 60 \delta_{i, 0}^{*}$, a shape factor of 1.4 is reached, typical for a turbulent boundary layer. For the turbulent interaction, only a small jump in the shape factor is observed from 1.35 to 1.67 at the shock impingement location, which implies that the turbulent boundary layer is still far removed from separation. The boundary layer shows a steady recovery, and within $80 \delta_{i, 0}^{*}$, the shape factor has returned to virtually the same level as was observed upstream of the interaction.

\section{Conclusions}

High-resolution PIV measurements were performed on an oblique shock wave reflection with an incoming laminar, transitional or turbulent boundary layer. The freestream flow conditions correspond to a Mach number of 1.7 and
Fig. 23 Development of the incompressible shape factor throughout a laminar, transitional or turbulent interaction. a Shape factor of the full boundary layer $H_{i}$, $\mathbf{b}$ shape factor of the boundary layer developing over the separation bubble $H_{i \text {,aw }}$

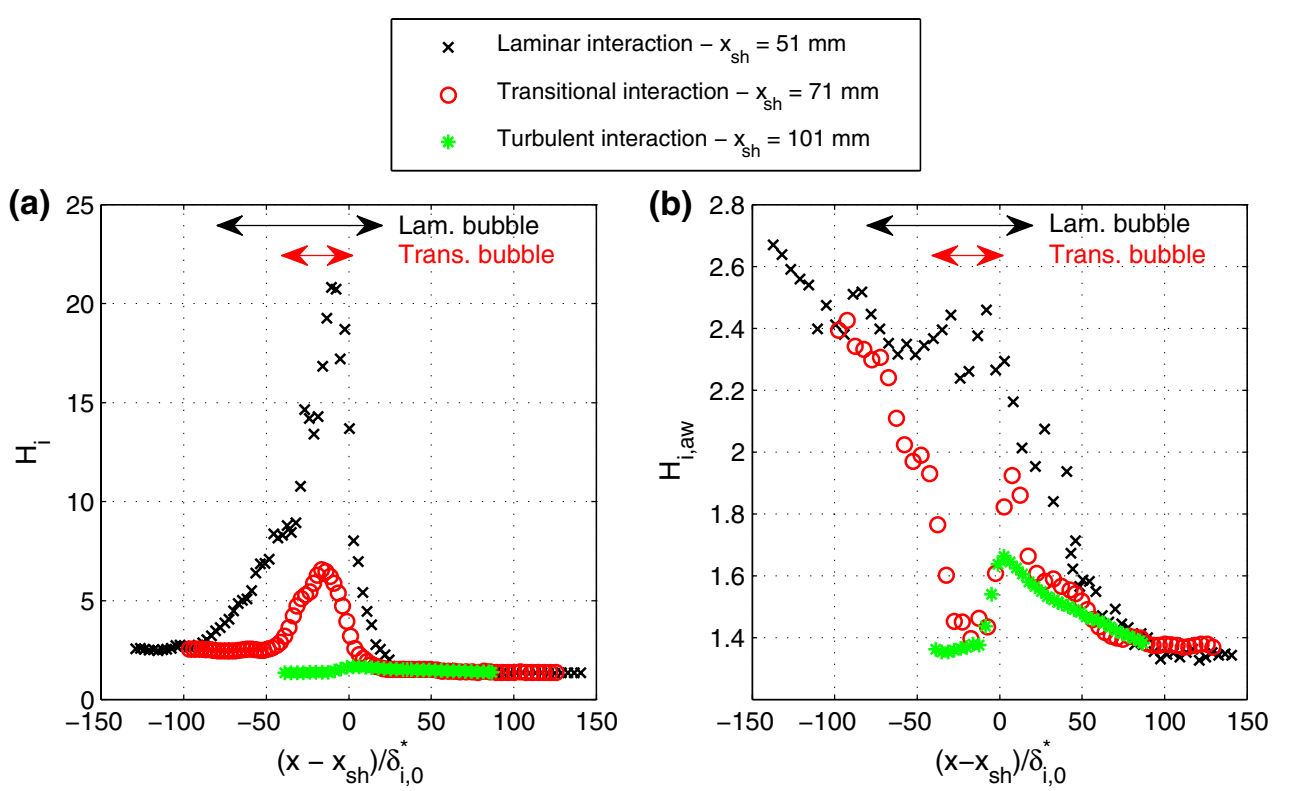


a unit Reynolds number of $35 \times 10^{6} \mathrm{~m}^{-1}$, while the shock generator was set to a flow deflection angle of $3^{\circ}$. The measurements were supported by oil-flow and high-speed Schlieren visualizations. Hot-wire measurements were performed to quantify the freestream turbulence level (mass flux RMS $0.77 \%$ ), and the fluctuations most critical for transition were found to be mainly acoustic of nature.

First, PIV measurements were performed on the undisturbed boundary layer to characterize the natural transition process. Seeding the thin 0.2-mm-thick laminar boundary layer proved to be highly challenging, since the $\mathrm{TiO}_{2}$ seeding particles migrate away from the wall near the plate leading edge. Consequently, virtually no seeding was present in the lower $\sim 40 \%$ of the boundary layer, making it only possible to measure the top $\sim 60 \%$ of the laminar boundary layer profile. Because of the relatively low seeding density in the boundary layer, the ensemble correlation technique (Meinhart et al. 2000) was used to extract the mean velocity field.

The seeding in the near-wall region greatly increases following transition; due to the effects of turbulent mixing, seeding particles are redistributed throughout the boundary layer. The seeding density in the near-wall region can therefore be used as an indicator for the state of the boundary layer. This was subsequently used to construct an approximate intermittency distribution, showing that the onset of transition is located around $x=55 \mathrm{~mm}\left(R e_{x}=1.9 \times 10^{6}\right)$ and that an intermittency level of $50 \%$ is reached around $x=71 \mathrm{~mm}\left(\operatorname{Re}_{x}=2.5 \times 10^{6}\right)$. These values are in good agreement with the results from the spark-light Schlieren and oil-flow visualizations.

The intermittency distribution was used to improve the quality of the transitional velocity profiles resulting from the ensemble correlation approach. The data set was divided in subsets of assumed laminar and turbulent images, based upon the particle density in the near-wall region of the image. The sets are independently analysed by ensemble correlation and afterwards recombined using weighted averaging. The resulting mean velocity field shows a smooth transition from a laminar to turbulent velocity profile over a streamwise length of $\sim 35 \mathrm{~mm}$.

The results from the boundary layer study were used to position the oblique shock wave in the laminar $\left(x_{\mathrm{sh}}=51 \mathrm{~mm}, R e_{x}=1.8 \times 10^{6}\right)$, transitional $\left(x_{\mathrm{sh}}=71 \mathrm{~mm}\right.$, $\left.R e_{x}=2.5 \times 10^{6}\right)$ or turbulent $\left(x_{\mathrm{sh}}=101 \mathrm{~mm}\right.$, $\left.R e_{x}=3.5 \times 10^{6}\right)$ region of the boundary layer. For the laminar interaction, a large reversed flow region was recorded, which spans $8.9 \mathrm{~mm}\left(96 \delta_{i, 0}^{*}\right)$ and reaches a maximum height of $0.21 \mathrm{~mm}\left(2.3 \delta_{i, 0}^{*}\right)$ in close proximity of the inviscid shock impingement location. The reversed flow region has a triangular shape and extends mostly upstream $(7.5 \mathrm{~mm})$ of the incident shock wave. By analysing the incompressible shape factor development over the reversed flow region, it is furthermore observed that in this case the incoming boundary layer remains in a laminar state up to the shock impingement location. Downstream of this location a rapid transition is observed and within $\sim 90 \delta_{i, 0}^{*}$ a fully turbulent profile is reached.

The transitional interaction $\left(x_{\mathrm{sh}}=71 \mathrm{~mm}\right)$ shows a smaller reversed flow region of $4.3 \mathrm{~mm}\left(43 \delta_{i, 0}^{*}\right)$ in streamwise direction. The maximum height of the reversed flow region is reached $1.1 \mathrm{~mm}\left(11 \delta_{i, 0}^{*}\right)$ upstream of the shock impingement location and equals $0.087 \mathrm{~mm}\left(0.87 \delta_{i, 0}^{*}\right)$. The boundary layer right upstream of the separation bubble is in a transitional state. The process of transition continues (and is accelerated) when the boundary layer is lifted over the separation bubble, and a turbulent state is reached right upstream of the shock impingement location.

The turbulent interaction $\left(x_{\mathrm{sh}}=101 \mathrm{~mm}\right)$ shows no separation under these conditions. This is in agreement with the free-interaction theory (Chapman et al. 1958), which predicts that a pressure ratio of at least 1.77 would be necessary to separate the turbulent incoming boundary layer, which is significantly higher than the ratio of 1.35 imposed by the oblique shock reflection in the present case.

Acknowledgments This work is supported by the European FP7 project TFAST (Grant Agreement No. 265455), which studies the effects of boundary layer transition on SWBLI. The authors also gratefully acknowledge the other TFAST partners for their shared insights and knowledge. The authors are furthermore grateful to Kyle Lynch for his support with processing the PIV data.

Open Access This article is distributed under the terms of the Creative Commons Attribution 4.0 International License (http://creativecommons.org/licenses/by/4.0/), which permits unrestricted use, distribution, and reproduction in any medium, provided you give appropriate credit to the original author(s) and the source, provide a link to the Creative Commons license, and indicate if changes were made.

\section{References}

Astarita T (2007) Analysis of weighting windows for image deformation methods in PIV. Exp Fluids 43(6):859-872

Auton TR (1987) The lift force on a spherical body in a rotational flow. J Fluid Mech 183:199-218

Babinsky H, Harvey JK (2011) Shock wave-boundary-layer interactions. Cambridge University Press, Cambridge

Bestion D, Gaviglio J, Bonnet JP (1983) Comparison between constant-current and constant-temperature hot-wire anemometers in highspeed flows. Rev Sci Instrum 54(11):1513-1524

Chapman DR, Kuehn DM, Larson HK (1958) Investigation of separated flows in supersonic and subsonic streams with emphasis on the effect of transition. Technical Report NACA report 1356

Clemens NT, Narayanaswamy V (2014) Low-frequency unsteadiness of shock wave/turbulent boundary layer interactions. Annu Rev Fluid Mech 46:469-492

Dhawan S, Narasimha R (1958) Some properties of boundary layer flow during the transition from laminar to turbulent motion. J Fluid Mech 3(4):418-436 
Dolling DS (2001) Fifty years of shock-wave/boundary-layer interaction research: what next? AIAA J 39(8):1517-1531

Duan L, Choudhari MM, Wu M (2014) Numerical study of acoustic radiation due to a supersonic turbulent boundary layer. J Fluid Mech 746:165-192

Erdem E, Kontis K, Johnstone E, Murray NP, Steelant J (2013) Experiments on transitional shock wave-boundary layer interactions at Mach 5. Exp Fluids 54(10):1-22. doi:10.1007/ s00348-013-1598-z

Gadd GE, Holder DW, Regan JD (1954) An experimental investigation of the interaction between shock waves and boundary layers. Proc R Soc Lond A 226(1165):227-253

Giepman RHM, Schrijer FFJ, Van Oudheusden BW (2015) Infrared thermography measurements on a moving boundary layer transition front in supersonic flow. AIAA J. doi:10.2514/1.J053910

Groot KJ, Pinna F, Van Oudheusden BW (2014) On closing the streamwise BiGlobal stability problem: the effect of boundary conditions. In: IUTAM-ABCM symposium on laminar-turbulent transition, Rio de Janeiro, Brazil

Hakkinen RJ, Greber I, Trilling L, Abarbanel SS (1959) The interaction of an oblique shock wave with a laminar boundary layer. NASA Memo 2-18-59W

Illingworth CR (1950) Some solutions of the equations of flow of a viscous compressible fluid. Proc Camb Philos Soc 46:469-478

Katzer E (1989) On the lengthscales of laminar shock/boundary layer interaction. J Fluid Mech 206:477-496

Laufer J (1961) Aerodynamic noise in supersonic wind tunnels. J Aerosp Sci 28(9):685-692

Laufer J (1964) Some statistical properties of the pressure field radiated by a turbulent boundary layer. Phys Fluids 7(8):1191-1197

Le Balleur J-C, Délery J (1973) Etude expérimentale de l'effet de la réflexion d'une onde de choc sur la transition de la couchelimite. Congrès Français de Mécanique, Poitiers (France), 17-20 Sept 1973

Lees L, Reeves BL (1964) Supersonic separated and reattaching laminar flows: I. General theory and application to adiabatic boundary-layer/shock-wave interactions. AIAA J 2:1907-1920
Mayle RE (1991) The role of laminar-turbulent transition in gas turbine engines. J Turbomach 113(4):509-536

Meinhart CD, Wereley ST, Santiago JG (2000) A PIV algorithm for estimating time-averaged velocity fields. J Fluids Eng 122(June):285-289

Meunier P, Leweke T (2003) Analysis and treatment of errors due to high velocity gradients in particle image velocimetry. Exp Fluids 35(5):408-421

Narasimha R (1957) On the distribution of intermittency in the transition region of a boundary layer. J Aeronaut Sci 24:711-712

Raffel M, Willert CE, Wereley ST, Kompenhans J (2007) Particle image velocimetry: a practical guide. Springer, Berlin

Ragni D, Schrijer FFJ, Van Oudheusden BW, Scarano F (2010) Particle tracer response across shocks measured by PIV. Exp Fluids 50(1):53-64

Samimy M, Lele SK (1991) Motion of particles with inertia in a compressible free shear layer. Phys Fluids A 3(8):1915-1923

Sansica A, Sandham ND, Hu ZW (2014) Forced response of a laminar shock-induced separation bubble. Phys Fluids 26(093601):1-14

Scarano F (2008) Overview of PIV in supersonic flows. In: Schroeder A, Willert CE (eds) Particle image velocimetry, topics in applied physics, vol 112, pp 445-463

Scarano F, Riethmuller ML (2000) Advances in iterative multigrid PIV image processing. Exp Fluids 29:S51-S60

Scharnowski S, Hain R, Kähler CJ (2012) Reynolds stress estimation up to single-pixel resolution using PIV-measurements. Exp Fluids 52:985-1002

Teramoto S (2005) Large-eddy simulation of transitional boundary layer with impinging shock wave. AIAA J 43(11):2354-2363

Theunissen R, Scarano F, Riethmuller ML (2008) On improvement of PIV image interrogation near stationary interfaces. Exp Fluids 45:557-572

Van Driest ER (1951) Turbulent boundary layer in compressible fluids. J Aeronaut Sci 18:145-160

Westerweel J, Scarano F (2005) Universal outlier detection for PIV data. Exp Fluids 39(6):1096-1100 